\title{
a does not imply the existence of a Suslin tree
}

\author{
Mirna Džamonja \\ Mathematics Department \\ University of Wisconsin-Madison \\ Madison, WI 53706, USA \\ dzamonja@math.wisc.edu \\ Saharon Shelah \\ Mathematics Department \\ Hebrew University of Jerusalem \\ 91904 Givat Ram, Israel \\ shelah@sunset.huji.ac.il
}

February 8, 2008

\begin{abstract}
We prove that $\mathbf{s}$ does not imply the existence of a Suslin tree, so answering a question of I. Juhász. 円
\end{abstract}

\footnotetext{
${ }^{1}$ The authors would like to thank the Israel Academy of Sciences and Humanities for a partial support through the Basic Research Foundation Grant number 0327398. Mirna Džamonja would in addition like to thank the Hebrew University of Jerusalem and the Lady Davis Foundation for the Forchheimer Postdoctoral Fellowship for the academic year 1994/95, when a part of the research for this paper was done. We also thank Rutgers University, as a part of the research for the paper was done in November 1995 while S. Shelah was visiting Rutgers University and M. Džamonja came for a short visit there. This publication is denoted [DjSh 604] in the list of publications of Saharon Shelah. The present address of M. Džamonja is: School of Mathematics, University of East Anglia, Norwich NR4 7TJ, England.
} 


\section{Introduction}

In his paper [Ost], A. Ostaszewski introduced the combinatorial principle $\mathbf{s}$. The principle is a weaker simple relative of $\diamond$, and it found many applications in set-theoretic topology, see $\mathrm{KuVa}$.

Definition 1.1. 9 means that there is a sequence $\left\langle A_{\delta}: \delta\right.$ limit $\left.<\omega_{1}\right\rangle$ such that

(i) Each $A_{\delta}$ is an unbounded subset of $\delta$ and

(ii) For every $A \in\left[\omega_{1}\right]^{\aleph_{1}}$, there is $\delta$ such that $A_{\delta} \subseteq A$. (Equivalently: there are stationarily many such $\delta$ ).

It is clear that $\diamond \Longrightarrow \mathbf{p}$, and it was already noticed in Ost that $\mathbf{p}+C H$ implies $\diamond$ (as explained in Ost], the argument is due to K. Devlin ( in Sh 98] also Burgess is credited)). For a while, it was open if $\boldsymbol{\beta}$ and $\diamond$ were actually equivalent, but this was settled by S. Shelah in [Sh 98, where a model of $\mathbf{s}$ is constructed in which $C H$ does not hold. The proof starts with $V=L$ (or just $\left.V \vDash C H+\diamond\left(\omega_{2}\right)\right)$, and $\aleph_{3}$ Cohen subsets of $\omega_{1}$ are added. Then $\aleph_{1}$ is collapsed, and it is shown that essentially, $\diamond\left(\omega_{2}\right)^{V}$ can serve as a $\mathbf{p}$-sequence in the final model.

Subsequently J. Baumgartner in an unpublished note gave a different construction of a model of $\mathbf{p}+\neg C H$, in which $\aleph_{1}$ is not collapsed. P. Komjáth [Ko], continuing the proof in Sh 98] proved it consistent to have $M A$ for countable partial orderings $+\neg C H$, and s. Then S. Fuchino, S. Shelah and L. Soukup [FShS 544 proved the same, without collapsing $\aleph_{1}$.

Having concluded that the principles, $\diamond$ and $\boldsymbol{p}$, are different we still may ask to which extent the consequences of $\diamond$ may be obtained from a. So, I. Juhász asks in [Mi]: "Does \& imply the existence of a Suslin tree?" This question is very natural, as $\diamond$ was formulated by Jensen in De in order to present his proof that there are Suslin trees in $L$. In addition, the existence of a Suslin tree is a long established test problem for various combinatorial principles to agree with.

Here we answer Juhász's question negatively. 
The idea of the proof is to start with a model of $\diamond+2^{\aleph_{1}}=\aleph_{2}$, and iterate a forcing which specializes Suslin trees, in an iteration of length $\omega_{2}$. Our plan is, similarly to Sh 98, to witness $\$$ by using $\diamond$ from the ground model in an essential way. Note that adding $\aleph_{1}$ Cohen reals destroys any club sequence from the ground model, which rules out finite support iterations.

Let $\chi$ be a large enough regular cardinal, and let $<_{\chi}^{*}$ be a fixed well order of $H(\chi)$. The formulation of $\diamond$ that we use is that there is a sequence

$$
\bar{N}^{*}=\left\langle\bar{N}^{\delta}=\left\langle N_{i}^{\delta}: i<\delta\right\rangle: \delta<\omega_{1}\right\rangle
$$

where each $\bar{N}^{\delta}$ is a continuously increasing sequence of countable elementary submodels of $\mathfrak{A} \stackrel{\text { def }}{=}\left(H(\chi), \in,<_{\chi}^{*}\right)$ with $N_{i}^{\delta} \cap \omega_{1}<\delta$ for $i<\delta$, and $\bar{N}^{*}$ is such that for every continuously increasing sequence $\bar{N}=\left\langle N_{i}: i<\omega_{1}\right\rangle$ of countable elementary submodels of $\mathfrak{A}$, there is a stationary set of $\delta<\omega_{1}$ such that the isomorphism type of $\bar{N}^{\delta}$ is the same as that of $\left\langle N_{i}: i<\delta\right\rangle$.

Let $P$ denote our forcing order. To show that holds in $V^{P}$, we show that for every condition $p \in P$, name $\underset{\sim}{\tau}$ such that $p \Vdash ~ " \tau \in \underset{\sim}{\mathcal{L}} \in\left[\omega_{1}\right]^{\aleph_{1}}$ ", and a sequence $\bar{N}$ as above with $p, \tau \in N_{0}$, there is a club of $\delta<\omega_{1}$ for which there is an unbounded sequence $\bar{\beta}_{\bar{N} \uparrow \delta}=\left\langle\beta_{k}: k<\omega\right\rangle \in V$ of ordinals below $\delta$, and a condition $r^{\oplus} \geq p$ such that $r^{\oplus} \Vdash "\left\{\beta_{k}: k<\omega\right\} \subseteq \mathcal{\sim}$ ". Moreover, the choice of $\left\{\beta_{k}: k<\omega\right\}$ and the fact that $r^{\oplus}$ exists, only depend on the isomorphism type of $\left\langle N_{i}: i<\delta\right\rangle$. Hence, if such a $\delta$ also has the property that the isomorphism type of $\left\langle N_{i}: i<\delta\right\rangle$ is the same as that of $\bar{N}^{\delta}$, then $\left\{\beta_{k}: k<\omega\right\}$ are definable from $\bar{N}^{\delta}$. So, the sequence $\left\langle A_{\delta}: \delta\right.$ limit $\left.<\omega_{1}\right\rangle$ given by

$$
A_{\delta} \stackrel{\text { def }}{=} \begin{cases}\operatorname{Rang}\left(\bar{\beta}_{\bar{N}^{\delta}}\right) & \text { if } \bar{\beta}_{\bar{N}^{\delta}} \text { is defined } \\ \delta & \text { otherwise }\end{cases}
$$

is a $\mathbf{p}$-sequence in $V^{P}$. A typical consideration to make is the following. Suppose that $p$ and $\tau$ are as above, while $\bar{N}=\left\langle N_{n}: n\langle\omega\rangle\right.$ is an increasing sequence of countable $\prec \mathfrak{A}$, with $N_{n} \in N_{n+1}$ for $n<\omega$ and $P, p, \tau \in N_{0}$, and we wish to construct $\bar{\beta} \stackrel{\text { def }}{=} \bar{\beta}_{\bar{N}}$ and $r^{\oplus}$ as above. Let $N_{\omega} \stackrel{\text { def }}{=} \bigcup_{n<\omega} N_{n}$ and $\delta \stackrel{\text { def }}{=} N_{\omega} \cap \omega_{1}$. We can find $r^{*} \geq p$ and $\beta^{*} \in \underset{\sim}{\tau}$ such that $r^{*} \Vdash$ " $\beta^{*} \in \underset{\sim}{\tau}$ ". Now, we can reflect $r^{*}$ and $\beta^{*}$ along $\bar{N}$, and so obtain sequences $\left\langle r_{n}: n<\omega\right\rangle$ and $\left\langle\beta_{n}: n<\omega\right\rangle$ such that $r_{n} \Vdash$ " $\beta_{n} \in \mathcal{\sim}$ ", while $\bigcup_{n<\omega} \beta_{n}=\delta$ and each $r_{n} \geq p$. 
If we can then find $r^{\oplus}$ as a common upper bound to $\left\{r_{n}: n<\omega\right\}$, we are done.

From what we said so far, our concerns are twofold: to have a forcing in which a certain amount of completeness is present, and on the other hand, to have a control of the way the reals are added (of course, we need to add reals, as we need to violate $\diamond)$. In the direction of our second aim, we divide the iteration in $E V E N$ and $O D D$ stages, and at the $E V E N$ stages we add a real which dominates all the reals in the previous model. In $O D D$ stages we do a forcing $N N R(T)$ which specializes an Aronszajn tree $T$, doing so without adding reals. Our forcing at $O D D$ stages is from $\mathrm{S}$. Shelah's [Sh -f, V $\S 6]$. At $E V E N$ stages, we use the forcing $U M$ for adding a universal meager set introduced by J. Truss in $[\mathrm{Tr}]$ (there it was called "amoeba forcing for category"), and used in S. Shelah's [Sh 176]. This forcing adds a dominating real. The forcing is $c c c$ in a strong way, and, as shown by Shelah in Sh 176. it has a strong completeness property, so called sweetness. This in particular implies that there is a dense set $\mathcal{D}$ of conditions in $U M$ on which there are equivalence relations $\left\langle E_{n}: n<\omega\right\rangle$, such that if a sequence $\bar{p}=\left\langle p^{n}: n \leq \omega\right\rangle$ from $\mathcal{D}$ has the property that $p^{n} E_{n} p^{\omega}$ for all $n$, then there is an upper bound to $\bar{p}$. A forcing notion with this property is called a sweetness model (see $\hat{2} 2$ for a better definition, and [Sh 176] for a real discussion). Our problem with completeness is then addressed by the way the iteration is done: we iterate with countable supports, but allow a condition $p_{1}$ to extend a condition $p_{0}$ only if the set of $E V E N$ coordinates in the $\operatorname{Dom}\left(p_{0}\right)$ on which $p_{1}$ differs from $p_{0}$ is finite (see [Sh -f, XIV] for a general treatment of such iterations and further references. An example of a such an iteration used in connection with os is in DjSh 574). Basically because at our EVEN stages we are doing a ccc forcing, and adding a dominating real, we can afford to do such an iteration and still end up with a proper forcing.

Now consider again $p$ and $r^{*}$ from our above described scenario. Before choosing $r^{*}$, we can construct increasing sequences $\bar{p}=\left\langle p_{n}: n<\omega\right\rangle$ and $\left\langle q_{n}: n<\omega\right\rangle$ which are sufficiently generic, in the following sense. We start with $p_{0}=p$, and choose $p_{n}$ and $q_{n}$ by induction on $n$. We shall have that $p_{n+1}$ and $p_{n}$ agree on $E V E N$ coordinates (we say $p_{n} \leq_{\text {pr }} p_{n+1}$ ), while $q_{n} \geq p_{n}$ 
and they agree on the $O D D$ coordinates, and $\operatorname{Dom}\left(p_{n}\right)=\operatorname{Dom}\left(q_{n}\right)$ (we say $\left.p_{n} \leq_{\text {apr }} q_{n}\right)$. During the induction, we make sure that for every formula $\varphi$ with parameters in $N_{\omega}$, there are infinitely many $n$ such that, given $p_{n}$, if we could have chosen $p_{n+1}$ and $q_{n+1}$ so that $\varphi\left(p_{n+1}, q_{n+1}\right)$ holds and the above description is not violated, then we have done so. We can also make sure that $p_{n}$ 's don't increase too much (for this we need to use dominating reals added by UM's along the way, and the way the iteration is defined), and thanks to a completeness-style property of $N N R(T)$-forcing this allows us to, at the end of this induction define $p_{\omega}$ as the limit of all $p_{n}$. Now we can take $r^{*} \geq p_{\omega}$. We can find a condition $p^{*}$ such that $p_{\omega} \leq_{\mathrm{pr}} p^{*} \leq_{\text {apr }} r^{*}$, and we can arrange so that the only odd coordinates on which $p^{*}$ and $r^{*}$ differ, are those in $\operatorname{Dom}\left(p_{\omega}\right)$.

The set $v_{0}$ of $E V E N$ coordinates in the domain of $p_{\omega}$ where $r^{*}$ and $p_{\omega}$ differ is finite, so is contained in $N_{n_{0}}$ for some $n_{0}<\omega$. The idea now is that $\left\langle r_{k}: k<\omega\right\rangle$ will be a subsequence $\left\langle q_{n_{k}}: k<\omega\right\rangle$ of $\left\langle q_{n}: n<\omega\right\rangle$, constructed by induction on $k$. By exhibiting at every stage $k$ a suitable formula $\varphi_{k}$ with parameters in $N_{\omega}$, such that $\varphi(x, y)$ densely holds for $x \geq_{\text {pr }} p_{n_{k}}$ and $y \geq$ apr $x$, we shall be able to control various properties of $r_{k}$ 's. For example, we'll be able to say that $q_{n_{k+1}} \Vdash$ " $\beta_{k+1} \in \mathcal{\sim}$ " for some $\beta_{k+1} \geq N_{n_{k}} \cap \omega_{1}$. Due to the nature of the $N N R(T)$ forcing and the preparations we made so far, we reduce the problem of $\left\langle r_{k}: k<\omega\right\rangle$ having an upper bound, basically to the problem of the projections of $q_{k}$ 's onto $v_{0}$ having an upper bound. However, this is not exactly what happens, because these projections are not necessarily conditions in $P$.

Given any condition $x$ in $P$, if we consider all the conditions $y$ such that $y \geq_{\text {apr }} x$, we obtain a sweetness model, $R_{x}$ (we really use a variation called $R_{x}^{+}$). We shall aim at a condition $r^{\prime} \in R_{p_{\omega}}$ such that $q_{n_{k}} E_{k} r^{\prime}$, where $E_{k}$ stands for the $k$-th equivalence relation in the sweetness model $R_{p w}$. Then we can use sweetness to assure that there is an upper bound as desired. What do we use as $r^{\prime}$ ?

The condition we would really like to use as $r^{\prime}$ is $r^{*} \uparrow \operatorname{Dom}\left(p_{\omega}\right)$. However, there are possibly coordinates of $r^{*}$ which are less than $\sup \left(N_{\omega} \cap \omega_{2}\right)$ and not in $N_{\omega}$, and names of $r^{*}(\gamma)$ for $\gamma \in \operatorname{Dom}\left(p_{\omega}\right)$ might depend on these "ghost" 
coordinates. So $r^{*} \uparrow \operatorname{Dom}\left(p_{\omega}\right)$ might not be a condition after all. Hence we have a task of finding a $r^{\prime} \in R_{p_{\omega}}$, which resembles $r^{*}$ sufficiently, and let $r_{k}$ 's be more and more equivalent to this $r^{\prime}$. However, we also have to be sure that our $r_{k}$ 's will be able to say something about $\beta^{*}$, to deliver the goods we implored them for.

We now place the entire situation in another countable elementary submodel of $\mathfrak{A}$, called $M$. We construct an increasing sequence $\bar{s}=\left\langle s_{n}: n<\omega\right\rangle$ sufficiently generic for $M$, starting with $s_{0}=p^{*}$, and requiring $s_{n}$ to only differ from $p^{*}$ on the coordinates outside of $N_{\omega}$. We let $r^{\prime}$ be whatever $\bar{s}$ forces $r^{*}$ to be inside of the $\operatorname{Dom}\left(p_{\omega}\right)$, i.e. $r^{\prime}=r^{*} / \bar{s}$ (see $\oint 8$ for a more precise definition). As $s_{n}$ 's were chosen to be sufficiently generic, we'll have that the naturally defined join of $s_{n}$ and $r^{\prime}$ will have the same $n$-th equivalence class in $R_{p^{*}}$ as $r^{\prime}$ does in $R_{p_{\omega}}$, for all large enough $n$. In $\$ 6$ we develop a method of saying this through a first order formula. Note that this join still contains the relevant information about $\beta^{*}$. So, using again the genericity of $\left\langle p_{n}: n<\omega\right\rangle$ we are done.

Swept under the rug in the above discussion is the fact that all the choices that we make have to be made depending only on the isomorphism type on $\bar{N}$, but this is easily arranged thanks to the well ordering of $H(\chi)$.

Taken with a grain of salt, as no proofs were given of our claims so far, and as introductions are usually easier to understand once whatever they are supposed to introduce is already understood, the above explanation might have convinced the reader that what we do is sufficient to prove the desired theorem. But is all this machinery really necessary? We can only say that we tried several other approaches, and the difficulty that we would face in general, is that some amount of completeness was missing. Such completeness in the present proof is achieved through the use of sweetness. One could presumably obtain a simpler proof that some different version of $\mathbf{p}$ does not imply the existence of a Suslin tree. Saharon Shelah has notes in which a version of the order from [BMR] is iterated with supports similar to the ones we are using, and the iteration shows that a weak version of $\mathbf{s}$ does not imply the existence of a Suslin tree. However, by the results in DjSh 574, this version of $\boldsymbol{s}$ is strictly weaker than 
The paper is organized as follows. In \$2 we give some background to $N N R(T)$ and $U M$. In $\S 3$ we state the Theorem. In $\S$ 团 we describe the iteration and prove various facts about it. Section $\$ 5$ contains the proof of the properness of the forcing used. In $\$ 6$ we give some definitions which are used to adapt the notion of sweetness to our situation. However, the notions from [Sh 176] have to be reformulated to fit our needs, hence in particular

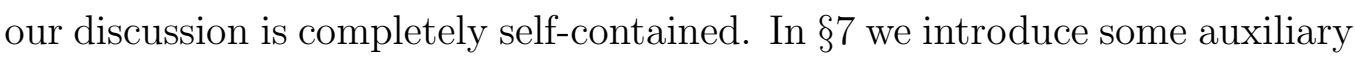
partial orders, and set ground for the proof in $\$ 8$. The main point of the proof is to obtain $\&$ in $V^{P}$. This argument is presented in $\delta 8$.

\section{Background}

The forcing needed in Section 3 will be an iteration of two kinds of individual forcings. The first is the forcing from [Sh -f, V§6], which specializes an Aronszajn tree $T$ without adding reals. We shall refer to this forcing as to $N N R(T)$. The other individual forcing is $U M$ ("universal meager"), the forcing introduced in [Tr] and used in [Sh 176, §7]. In this section we review some properties of these forcings that will be needed for the proof in $\$ 3-\$ 8$.

Notation 2.1. (1) For two sequences $\bar{s}$ and $\bar{t}$, we say that $\bar{s} \cap \bar{t}=\emptyset$ whenever the ranges of $\bar{s}$ and $\bar{t}$ are disjoint.

(2) $\mathbb{Q}$ stands for the rational numbers with their usual ordering.

(3) If $T$ is a tree, then $<_{T}$ denotes the tree order of $T$. For $x \in T$, we let $\operatorname{ht}_{T}(x) \stackrel{\text { def }}{=} \operatorname{otp}\left(\left\{y: y<_{T} x\right\}\right)$. We may omit $T$ in this notation, if the $T$ we mean is clear from the context.

If $T$ is an $\omega_{1}$-tree and $i<\omega_{1}$, then $T_{i}$ denotes the $i$-level of $T$, i.e. $\{x \in T: \operatorname{ht}(x)=i\}$.

If $\bar{x}$ and $\bar{y}$ are two sequences of elements of $T$, then $\bar{x}<_{T} \bar{y}$ means that $\bar{x}$ and $\bar{y}$ have the same length, and for every $l \in \operatorname{Dom}(x)$, we have $x_{l}<_{T} y_{l}$.

If $\operatorname{Rang}(\bar{x}) \cap \operatorname{Rang}(\bar{y})=\emptyset$, we say that $\bar{x}$ and $\bar{y}$ are disjoint.

(4) If $\eta$ and $\rho$ are sequences, then $\eta \triangleleft \rho$ means that $\eta$ is an initial segment of $\rho$.

(5) Without loss of generality, all Aronszajn trees $T$ that we mention will be assumed to have the property that $T_{\alpha} \subseteq[\omega \alpha, \omega(\alpha+1))$, for all $\alpha<\omega_{1}$. In 
addition, we'll assume $\left|T_{\alpha}\right|=\aleph_{0}$ for all $\alpha<\omega_{1}$. As we might want to consider subtrees of $T$, we do not assume necessarily that $T_{\alpha}=[\omega \alpha, \omega(\alpha+1))$ for all $\alpha$.

(6) If $T$ is an Aronszajn tree and $\alpha<\omega_{1}$, then $\left\{x_{l}^{T_{\alpha}}: l<\omega\right\}$ is the increasing enumeration of $T_{\alpha}$.

(7) Suppose that $T$ is an Aronszajn tree and $m<\omega$, while $\alpha<\omega_{1}$. We define

$$
w_{m}^{T_{\alpha}} \stackrel{\text { def }}{=}\left\{x_{l}^{T_{\alpha}}: l<m\right\} .
$$

(8) We often identify a node $x \in T_{\delta}$ for limit $\delta$ with the branch $\left\{y: y<_{T} x\right\}$. Also, if $\alpha<\beta$ and $x \in T_{\beta}$, then $x \uparrow(\alpha+1)$ denotes the unique $y \in T_{\alpha}$ with $y<_{T} x$.

Definition 2.2. Given an Aronszajn tree $T$, we define (1)

$$
\begin{aligned}
N N R_{1}(T) \stackrel{\text { def }}{=}\{(f, C): \quad & C \text { is a closed subset of some } \alpha+1<\omega_{1} \\
& \text { with the last element } \alpha \stackrel{\text { def }}{=} \operatorname{lt}(C), \text { and } \\
& \left.f: \bigcup_{i \in C} T_{i} \rightarrow \mathbb{Q} \text { is monotonically increasing }\right\} .
\end{aligned}
$$

For $\left(f_{1}, C_{1}\right)$ and $\left(f_{2}, C_{2}\right)$ in $N N R_{1}(T)$, we say $\left(f_{1}, C_{1}\right) \leq_{N N R_{1}(T)}\left(f_{2}, C_{2}\right)$ iff $C_{1}=C_{2} \uparrow\left(\operatorname{lt}\left(C_{1}\right)+1\right)$ and $f_{1} \subseteq f_{2}$.

(2) $\Gamma$ is a $T$-promise $\underline{\text { iff }}$ there is a club $C(\Gamma)$ of $\omega_{1}$ and $n=n(\Gamma)<\omega$ such that:

(a) All elements of $\Gamma$ have form $\left\langle x_{0}, \ldots, x_{n-1}\right\rangle$ where $\left\langle x_{0}, \ldots, x_{n-1}\right\rangle$ are such that

$$
(\exists \alpha \in C(\Gamma))\left[(\forall i \neq j<n)\left(x_{i} \neq x_{j}\right) \&(\forall i<n)\left(x_{i} \in T_{\alpha}\right)\right]
$$

(b) If $\alpha<\beta \in C(\Gamma) \& \bar{x} \in \Gamma \bigcap^{n} T_{\alpha}$, then there are infinitely many pairwise disjoint $\bar{y} \in{ }^{n} T_{\beta}$ such that $\bar{x}<_{T} \bar{y}$.

(c) $\Gamma \bigcap^{n}\left(T_{\min (C(\Gamma))}\right) \neq \emptyset$. 
(3) $(f, C) \in N N R_{1}(T)$ fulfills a promise $\Gamma \underline{\text { iff }}$

$(\alpha) \operatorname{lt}(C) \in C(\Gamma)$ and $C(\Gamma) \supseteq C \backslash \min (C(\Gamma))$..

( $\beta$ ) For all $\alpha<\beta \in C(\Gamma) \cap C$, and for all $\bar{x} \in \Gamma \bigcap^{n(\Gamma)}\left(T_{\alpha}\right)$ the following holds:

$(\oplus)$ For all $\epsilon>0$, there are infinitely many pairwise disjoint $\bar{y} \in{ }^{n(\Gamma)} T_{\beta}$ with $\bar{x}<_{T} \bar{y}$ and such that for all $l<n(\Gamma)$ we have

$$
f\left(x_{l}\right)<f\left(y_{l}\right)<f\left(x_{l}\right)+\epsilon .
$$

The intention of fulfilling a promise is that $f$ is guaranteed not to grow too much along the relevant branches.

(4)

$$
\begin{aligned}
N N R(T) \stackrel{\text { def }}{=}\{(f, C, \Psi): \quad & (f, C) \in N N R_{1}(T) \text { and } \Psi \text { is a countable set } \\
& \text { of promises which }(f, C) \text { fulfills }\} .
\end{aligned}
$$

We let $\left(f_{1}, C_{1}, \Psi_{1}\right) \leq\left(f_{2}, C_{2}, \Psi_{2}\right)$ iff $\left(f_{1}, C_{1}\right) \leq_{N N R_{1}(T)}\left(f_{2}, C_{2}\right)$ and $\Psi_{1}$ is a subset of $\Psi_{2}$, while $C_{2} \backslash C_{1} \subseteq \bigcap_{\Gamma \in \Psi_{1}} C(\Gamma)$.

Notation 2.3. For $p=(f, C, \Psi) \in N N R(T)$, we write $f^{p} \stackrel{\text { def }}{=} f, C^{p} \stackrel{\text { def }}{=} C$, $\Psi^{p}=\Psi$ and $\operatorname{lt}(p) \stackrel{\text { def }}{=} \operatorname{lt}\left(C_{p}\right)$.

Definition 2.4. [Sh -f, VIII §2] Given $\kappa$ an infinite cardinal. A forcing notion $P$ is said to satisfy $\kappa$-pic* iff for all large enough $\chi$ and well orders $<_{\chi}^{*}$ of $H(\chi)$, we have:

Suppose that $i<j<\kappa$, and $N_{i}, N_{j} \prec \mathfrak{A}=\left(H(\chi), \in,<_{\chi}^{*}\right)$ are countable with $\kappa, P \in N_{i} \cap N_{j}$, while $N_{i} \cap \kappa \subseteq j$ and $N_{i} \cap i=N_{j} \cap j$, and $N_{l}$ is the Skolem hull in $\mathfrak{A}$ of $\left(N_{i} \cap N_{j}\right) \cup\{l\}$ for $l \in\{i, j\}$. Further suppose that $p \in P \cap N_{i}$, while $h: N_{i} \rightarrow N_{j}$ is an isomorphism with $h \uparrow\left(N_{i} \cap N_{j}\right)$ being the identity, and $h(i)=j$.

Then there is $q \in P$ such that 
(a) $p, h(p) \leq q$, and for every maximal antichain $I \subseteq P$ with $I \in N_{i}$, we have that $I \cap N_{i}$ is predense above $q$.

(b) For every $r \in N_{i} \cap P$ and $q^{\prime}$ such that $q \leq q^{\prime} \in P$, there is $q^{\prime \prime} \in P$ such that

$$
r \leq q^{\prime} \Longleftrightarrow h(r) \leq q^{\prime \prime}
$$

Fact 2.5. [Sh -f, VIII, 2.5* and 2.9*] Suppose that $\bar{Q}=\left\langle P_{\alpha}, Q_{\alpha}: \alpha<\alpha^{*}\right\rangle$ is a countable support iteration and $\kappa$ is regular. Further suppose that for each $\alpha<\alpha^{*}$ we have $\Vdash_{P_{\alpha}}$ " $Q_{\alpha}$ has $\kappa$-pic*." Then

(1) If $\alpha^{*}<\kappa$, then $P_{\alpha^{*}}$ satisfies $\kappa$-pic*

(2) If $\alpha^{*} \leq \kappa$ and $(\forall \mu<\kappa)\left(\mu^{\aleph_{0}}<\kappa\right)$, then $P_{\kappa}$ satisfies $\kappa-c c$.

(3) If $\alpha^{*}<\kappa$ and $(\forall \mu<\kappa)\left(\mu^{\aleph_{0}}<\kappa\right)$, then

$$
\Vdash_{P_{\alpha^{*}}} "(\forall \mu<\kappa)\left(\mu^{\aleph_{0}}<\kappa\right) " .
$$

Fact 2.6. [Sh -f, V§6] Suppose $V \models C H$. Then $N N R(T)$ is a proper $\aleph_{2}-c c$, moreover $\aleph_{2}$-pic ${ }^{*}$, forcing which specializes $T$ without adding reals.

Note that $|N N R(T)| \leq 2^{\aleph_{1}}$.

Fact 2.7. [Sh -f V, 6.7.] Suppose that $\chi$ is large enough and $N \prec(H(\chi), \in)$ is countable such that $T \in N$. Let $\delta \stackrel{\text { def }}{=} N \cap \omega_{1}$ and $\epsilon>0$. Further suppose that $p \in N N R(T) \cap N$ and for some $n<\omega$ we have $b_{0}, \ldots, b_{n-1}$ are distinct branches of $T_{\delta}$, while $I \in N$ is an open dense subset of $P$.

Then there is $q \geq p$ with $q \in I \cap N$, and such that for all $i<n$ we have

$$
f_{q}\left(b_{i}(\operatorname{lt}(q))\right)<f_{p}\left(b_{i}(\operatorname{lt}(p))\right)+\epsilon .
$$

The following is well known and follows from the above Fact 2.7: 
Claim 2.8. Suppose that $\chi$ is large enough and $N \prec(H(\chi), \in)$ is countable such that $T \in N$, while $p \in N N R(T) \cap N$. Then there is $q \geq p$ which is $(N, N N R(T))$-generic and $\operatorname{lt}(q)=N \cap \omega_{1}$.

Proof of the Claim. Let $\left\{I_{n}: n<\omega\right\}$ enumerate all open dense subsets of $N N R(T)$ which are elements of $N$. Using Fact 2.7, we can build an increasing sequence $\left\langle p_{n}: n<\omega\right\rangle$ of conditions in $N N R(T)$ such that

(a) $p_{0}=p$

(b) $p_{n} \in N$.

(c) For every $n<\omega$, for every $x \in w_{n+1}^{T_{1 \mathrm{t}\left(p_{n+1}\right)}}$ we have

$$
f_{p_{n+1}}(x)<f_{p_{n}}\left(x \uparrow \operatorname{lt}\left(p_{n}\right)\right)+1 / 2^{n}
$$

(d) $p_{n+1} \in I_{n}$.

Now we can define $q$ by letting $\operatorname{lt}(q) \stackrel{\text { def }}{=} \delta, C^{q} \stackrel{\text { def }}{=} \cup_{n<\omega} C^{p_{n}} \cup\{\delta\}$, while $f^{q} \stackrel{\text { def }}{=}$ $\bigcup_{n<\omega} f^{p_{n}} \cup\left\{\left(x, \sup _{n<\omega}\left(f^{p_{n}}\left(x \vdash\left[\operatorname{lt}\left(p_{n}\right)+1\right]\right)\right): x \in T_{\delta} \& x \uparrow\left[\operatorname{lt}\left(p_{n}\right)+1\right] \in \operatorname{Dom}\left(f^{p_{n}}\right)\right\}\right.$, and $\Psi^{q} \stackrel{\text { def }}{=} \bigcup_{n<\omega} \Psi^{p_{n}}$. It is easily seen that $q$ is as required. $\star 2.8$

Definition 2.9. (1) $\mathcal{T} \subseteq{ }^{<\omega_{2}}$ is a nowhere dense tree $\underline{\text { iff }}$ for all $\eta \in \mathcal{T}$, there is $\rho \in{ }^{<\omega} 2 \backslash \mathcal{T}$ with $\eta \triangleleft \rho$.

(2) $\mathcal{T} \subseteq{ }^{<\omega_{2}}$ is perfect iff for all $\eta \in \mathcal{T}$, there are $\rho_{1} \neq \rho_{2}$ both in $\mathcal{T}$ and both extending $\eta$.

$$
\begin{aligned}
U M \stackrel{\text { def }}{=}\{(t, \mathcal{T}): \quad & \mathcal{T} \subseteq{ }^{<\omega} 2 \text { is a perfect nowhere dense tree } \\
& \text { and for some } \left.n \text { we have } t=\mathcal{T} \cap{ }^{n} 2\right\} .
\end{aligned}
$$

For $\left(t_{1}, \mathcal{T}_{1}\right),\left(t_{2}, \mathcal{T}_{2}\right) \in U M$, we say $\left(t_{1}, \mathcal{T}_{1}\right) \leq\left(t_{2}, \mathcal{T}_{2}\right)$ iff for some $n$ we have $t_{1}=t_{2} \cap{ }^{n} 2$, and $\mathcal{T}_{1} \subseteq \mathcal{T}_{2}$. 
Fact 2.10. $[\operatorname{Tr}]$ Suppose that $G$ is $U M$-generic.

Then $S \stackrel{\text { def }}{=} \cup\{\mathcal{T}:(\exists t)((t, \mathcal{T}) \in G)\}$ is a nowhere dense subtree of $<\omega_{2}$.

The following consequence of Fact 2.10 is also well known:

Claim 2.11. $U M$ adds a real which dominates all the reals from the ground model.

Proof of the Claim. Let $S$ be the nowhere dense tree added by $U M$. We define $g_{S} \in{ }^{\omega} \omega$ by letting

$$
g_{S}(n) \stackrel{\text { def }}{=} \min \left\{m:\left(\forall \eta \in S \cap^{n} 2\right)\left(\exists \rho \in{ }^{m} 2 \backslash S\right)(\eta \triangleleft \rho)\right\} .
$$

Hence $g_{S}$ is well defined, and we shall now see that it dominates all $f \in{ }^{\omega} \omega$ of the ground model. Fix such an $f$, and note that the set of all conditions $(t, \mathcal{T})$ which satisfy

$$
\left(\exists n_{0}\right)\left(\forall n \geq n_{0}\right)\left[\min \left\{m:\left(\forall \eta \in^{n} 2\right)\left(\exists \rho \in^{m} 2 \backslash \mathcal{T}\right)(\eta \triangleleft \rho)\right\}>f(n)\right]
$$

is dense in $U M . \star 2.11$

Notation 2.12. For $p=(t, \mathcal{T}) \in U M$, we let $t^{p} \stackrel{\text { def }}{=} t$ and $\mathcal{T}^{p} \stackrel{\text { def }}{=} \mathcal{T}$.

Definition 2.13. [Sh 176, §7] (1) A forcing notion $P$ is sweet if there is a subset of $\mathcal{D}$ of $P$ and equivalence relations $E_{n}$ on $\mathcal{D}$ for $n<\omega$, such that

(a) $\mathcal{D} \subseteq P$ is dense, $E_{n+1}$ refines $E_{n}$ and $E_{n}$ has countably many equivalence classes.

(b) For every $n<\omega$ and $p \in \mathcal{D}$, the equivalence class $p / E_{n}$ is directed.

(c) If $p^{i} \in \mathcal{D}$ for $i \leq \omega$, and $p^{i} E_{i} p^{\omega}$, then $\left\{p^{i}: i \leq \omega\right\}$ has an upper bound; moreover, for each $n<\omega$ the set $\left\{p^{i}: n \leq i \leq \omega\right\}$ has an upper bound in $p^{\omega} / E_{n}$. 
(d) For every $p, q$ in $\mathcal{D}$ and $n<\omega$, there is $k<\omega$ such that for every $p^{\prime} \in p / E_{k}$,

$$
\left(\exists r \in q / E_{n}\right)(r \geq p) \Longrightarrow\left(\exists r \in q / E_{n}\right)\left(r \geq p^{\prime}\right) .
$$

(2) If (1) above holds, we say that $\left(P, \mathcal{D}, E_{n}\right)_{n<\omega}$ is a sweetness model.

Definition 2.14. $[$ Sh $176, \S 7]$ Suppose that $\mathfrak{B}=\left(P, \mathcal{D}^{0}, E_{n}^{0}\right)_{n<\omega}$ is a sweetness model and $\bar{A}=\left\langle A_{e}: e<\omega\right\rangle$ enumerates $\left\{p / E_{n}^{0}: n<\omega \& p \in \mathcal{D}^{0}\right\}$.

(1) For $q \in \mathcal{D}^{0}$ we define $k_{m}(q)$ as the minimal $k<\omega$ such that for every $q^{\prime} \in q / E_{k}^{0}$ we have that

$$
\left(\exists r \in A_{m}\right)(r \geq q) \Longleftrightarrow\left(\exists r^{\prime} \in A_{m}\right)\left(r^{\prime} \geq q^{\prime}\right) .
$$

(2) We define

$$
\mathcal{D} \stackrel{\text { def }}{=}\left\{(p,(t, \mathcal{T})): p \in \mathcal{D}^{0} \& \Vdash_{P} "(t, \mathcal{T}) " \in U U_{\sim} M "\right\} .
$$

For $n<\omega$ and $\left(p_{l},\left(t_{l}, \mathcal{T}_{\sim}\right)\right) \in P * U_{\sim} M(l=1,2)$, we say that

$$
\left(p_{1},\left(t_{1}, \mathcal{T}_{\sim}\right)\right) E_{n}\left(p_{2},\left(t_{2}, \mathcal{T}_{\sim}\right)\right)
$$

iff the following conditions hold:

( $\alpha) p_{1} E_{n}^{0} p_{2}$

( $\beta) t_{1}=t_{2}$

$(\gamma)$ for every $m<n$, there is $p \in A_{m}$ with $p \geq p_{1}$ iff there is $p \in A_{m}$ with $p \geq p_{2}$.

$(\delta)$ suppose that $m<n$ and there is $p \in A_{m}$ such that $p \geq p_{1}$, and let $\eta \in{ }^{<n} 2$. Then there is $p \in A_{m}$ such that $p \Vdash$ " $\eta \notin \mathcal{T}_{\sim}$ " iff there is $p \in A_{m}$ such that $p \Vdash " \eta \notin \underset{\sim}{\mathcal{T}}$ ",

$(\varepsilon)$ for all $m<n$ we have $k_{m}\left(p_{1}\right)=k_{m}\left(p_{2}\right)$ and for all $m<n$ we have $p_{1} E_{k_{m}\left(p_{1}\right)}^{0} p_{2}$. 
Definition 2.15. [Sh 176, §7] Sweetness models $\mathfrak{B}_{1} \stackrel{\text { def }}{=}\left(P^{1}, \mathcal{D}^{1}, E_{n}^{1}\right)_{n<\omega}$ and $\mathfrak{B}_{2} \stackrel{\text { def }}{=}\left(P^{2}, \mathcal{D}^{2}, E_{n}^{2}\right)_{n<\omega}$ are said to satisfy $\mathfrak{B}_{1}<\mathfrak{B}_{2}$ iff

(a) $P^{1}$ is a complete suborder of $P^{2}$, while $\mathcal{D}^{1} \subseteq \mathcal{D}^{2}$ and for each $n$ we have that $E_{n}^{1}$ is $E_{n}^{2}$ restricted to $\mathcal{D}^{1}$,

(b) For all $p \in \mathcal{D}^{1}$ and $n<\omega$ we have $p / E_{n}^{2} \subseteq P^{1}$,

(c) If $p \leq q$ and $q \in \mathcal{D}^{1}$, while $p \in \mathcal{D}^{2}$, then $p \in \mathcal{D}^{1}$.

Notation 2.16. Suppose that $\mathfrak{B}$ and $P$ are as in the assumptions of Definition 2.14 and $\mathcal{D}$ and $E_{n}(n<\omega)$ are as defined in Definition 2.14. We say that

$$
\mathfrak{B}_{\bar{A}} * U \stackrel{\sim}{\text { def }}=\left(P * U M, \mathcal{\sim}, E_{n}\right)_{n<\omega}
$$

is the canonical sweetness model on $P * U$ M with respect to $\mathfrak{B}$ and $\bar{A}$.

Fact 2.17. [The Composition Claim, Sh $176, \S 7$ ] If $\mathfrak{B}$ is a sweetness model and $\bar{A}$ is an enumeration of the equivalence classes of $\mathfrak{B}$, then $\mathfrak{B}_{\bar{A}} * U_{\sim} M$ is a

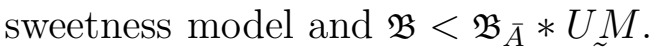

Fact 2.18. [Sh $176, \S 7$ ] Suppose that for $k<n$ we have that $\left(P^{k}, \mathcal{D}^{k}, E_{n}^{k}\right)_{n<\omega}$ is a sweetness model and

$$
\left(P^{k}, \mathcal{D}^{k}, E_{n}^{k}\right)_{n<\omega}<\left(P^{k+1}, \mathcal{D}^{k+1}, E_{n}^{k+1}\right)_{n<\omega} .
$$

Then $\left(\bigcup_{k<\omega} P^{k}, \bigcup_{k<\omega} \mathcal{D}^{k}, \bigcup_{k<\omega} E_{n}^{k}\right)_{n<\omega}$ is a sweetness model with the property that for all $k<\omega$ we have

$$
\left(P^{k}, \mathcal{D}^{k}, E_{n}^{k}\right)_{n<\omega}<\left(\bigcup_{k<\omega} P^{k}, \bigcup_{k<\omega} \mathcal{D}^{k}, \bigcup_{k<\omega} E_{n}^{k}\right)_{n<\omega} .
$$

Note 2.19. Any sweet forcing $P$ is $c c c$, even is $\sigma$-centered.

[Why? Let $\left\{A_{m}: m<\omega\right\}$ enumerate all $q / E_{n}$ for $q \in \mathcal{D}$ and $n<\omega$. For $m<\omega$, let $B_{m} \stackrel{\text { def }}{=}\left\{q:\left(\exists p \in A_{m}\right)(p \geq q)\right\}$, hence each $B_{m}$ is directed and $\left.P=\bigcup_{m<\omega} B_{m}.\right]$ 


\section{3 \& does not imply the existence of a Suslin tree}

Theorem 3.1. Assume that $V \models " \diamond\left(\omega_{1}\right)+2^{\aleph_{1}}=\aleph_{2}$ ".

Then there is a proper $\aleph_{2}-c c$ forcing notion $P$ such that $\Vdash_{P}$ " $\boldsymbol{\beta}+$ there are no Suslin trees".

The proof of this Theorem is presented in Sections $\oint$ 田 $\delta$.

\section{Forcing and Iteration}

Notation 4.1. " $\mathcal{T}$ is $N W D$ " means that $\mathcal{T}$ is a perfect nowhere dense subtree of $<^{\omega} 2$.

Definition 4.2. By simultaneous induction on $\alpha \leq \omega_{2}$, we define items (1)(3) and prove Claim 4.3 below.

(1)

$$
P_{\alpha} \stackrel{\text { def }}{=}\left\{\begin{array}{c}
(I) \operatorname{Dom}(p) \text { is a countable } \subseteq \alpha \\
p: \quad(I I) \text { For all } i \in \operatorname{Dom}(p) \text { we have } \\
\Vdash_{P_{i}} " p(i) \in Q_{i} "
\end{array}\right\} \text {. }
$$

(2) If $\alpha=2 i$ for some $i$, then $\Vdash_{P_{\alpha}}$ " $Q_{\alpha}=U M_{\sim}$ ",

(3) If $\alpha=2 i+1$ for some $i$, then $\Vdash_{P_{\alpha}}$ " $Q_{\alpha}=N N R\left(\underset{\sim}{T}{ }^{\alpha}\right)$ ", where $\underset{\sim}{T}$, is a $P_{2 i}$-name of an Aronszajn tree, handed to us by the bookkeeping (see Claim 4.3 below.) (We emphasize that ${\underset{\sim}{\alpha}}^{\alpha}$ is a $P_{2 i}$-name, not a $P_{\alpha}$-name.)

(4) We say that $p \leq q$ for $p, q \in P_{\alpha}$ iff for all $j \in \operatorname{Dom}(p)$ we have

(a) $j$ even $\Longrightarrow q \uparrow j \Vdash_{P_{j}} " p(j) \leq q(j)$ " and

(b) $\{2 i \in \operatorname{Dom}(p): \neg(q \uparrow(2 i) \Vdash " p(2 i)=q(2 i) ")\}$ is finite.

(c) $j$ odd $\Longrightarrow \vdash_{P_{j}}$ " $p(j) \leq q(j) "$. 
(Note that $\left(P_{\alpha}, \leq\right)$ is a forcing notion).

Claim 4.3. If $\alpha=2 i+1$ for some $i$, then

$$
\Vdash_{P_{\alpha}}{ }^{T}{ }^{\alpha} \text { is an Aronszajn tree ". }
$$

Proof of the Claim. This easily follows from the fact that $U M$ is $\sigma$ centered (Fact 2.17 and Note 2.19). Namely, suppose that $\alpha=2 i+1$. We know that

$$
\Vdash_{P_{2 i}} \text { "T } T^{\alpha} \text { is an Aronszajn tree", }
$$

as $\underset{\sim}{T}{ }^{\alpha}$ is a $P_{2 i}$-name of an Aronszajn tree. We only have to check that $Q_{2 i}$ does not add any uncountable branches to $\underset{\sim}{T}$. We work in $V^{P_{2 i}}$. Suppose $p \in U M$ and

$$
p \Vdash " \sim \mathcal{\sim}: \omega_{1} \rightarrow T^{\alpha} \text { is increasing } \&\left(\forall \gamma<\omega_{1}\right)\left(\mathcal{\sim}(\gamma) \in T_{\gamma}^{\alpha}\right) . "
$$

For $\gamma<\omega_{1}$, let $\mathcal{D}_{\gamma}$ be the set of conditions of $U M$ which are above $p$ and decide the value of $\underset{\sim}{\tau}(\gamma)$. Then there is a directed subset $A$ of $U M$ and an uncountable $B \subseteq \omega_{1}$ such that

$$
\gamma \in B \Longrightarrow A \cap \mathcal{D}_{\gamma} \neq \emptyset
$$

It follows from the directedness of $A$ that for all $\gamma \in B$, there is a unique $l_{\gamma}$ such that for all $q \in \mathcal{D}_{\gamma} \cap A$ we have $q \Vdash$ " $\mathcal{\sim}(\gamma)=x_{l_{\gamma}}^{T_{\gamma}^{\alpha}}$ ". Again by the directedness of $A$, if $\gamma_{1}<\gamma_{2} \in B$ we must have $x_{l_{\gamma_{1}}}^{T_{\gamma_{1}}^{\alpha}}<_{T^{\alpha}} x_{l_{\gamma_{2}}}^{T_{\gamma_{2}}^{\alpha}}$, a contradiction.

$\star 4.3$

Notation 4.4. (1) For $j<\alpha$ and $p, r \in P_{j}$ we say $p \leq_{\text {apr }} r$ iff

(i) $\operatorname{Dom}(p)=\operatorname{Dom}(r) \underline{\text { and }}$

(ii) $p \leq r \underline{\text { and }}$

(iii) $(\forall 2 k+1 \in \operatorname{Dom}(p))\left(r \uparrow(2 k+1) \Vdash_{P_{2 k+1}} " r(2 k+1)=p(2 k+1) "\right)$. 
(2) For $j<\alpha$ and $p, r \in P_{j}$ we say $p \leq_{\mathrm{pr}} r$ iff

(a) $p \leq r \underline{\text { and }}$

(b) $(\forall 2 k \in \operatorname{Dom}(p))\left(r \uparrow(2 k) \Vdash_{P_{2 k}} " r(2 k)=p(2 k) "\right)$.

(3) For $p \in P_{\alpha}$ we define $q=p \nmid E V E N$ by $\operatorname{Dom}(q) \stackrel{\text { def }}{=} \operatorname{Dom}(p) \cap E V E N$ and for $\beta \in \operatorname{Dom}(q)$, by letting $q(\beta) \stackrel{\text { def }}{=} p(\beta)$.

Observation 4.5. $(1) \leq_{\text {pr }}$ and $\leq_{\text {apr }}$ are partial orders.

(2) If $p \in P_{\alpha}$, then $p \nmid E V E N \in P_{\alpha}$ and $p \geq p\lceil E V E N$.

Definition 4.6. By simultaneous induction on $\alpha \leq \omega_{2}$, we define items (1)(4) below and prove Claim 4.7 below..$^{2}$

(1)

$$
P_{\alpha}^{\prime} \stackrel{\text { def }}{=}\left\{\begin{array}{c}
\text { (A) If } 2 i \in \operatorname{Dom}(p) \text {, then } \\
p(2 i) \text { is simple above } p \uparrow 2 i \\
p \in P_{\alpha}^{\prime}: \quad(B) \text { There is } \delta^{*}(p) \text { limit }<\omega_{1} \text { such that } \\
2 i+1 \in \operatorname{Dom}(p) \Longrightarrow \\
\Vdash_{P_{2 i+1}} \text { "lt }(p(2 i+1))=\delta^{*}(p) "
\end{array}\right\},
$$

with the order inherited from $P_{\alpha}$.

(2) For $2 i<\alpha$ and $p \in P_{2 i}$, we say that $p(2 i)=\left({\underset{\sim}{t}}^{2 i},{\underset{\sim}{\sim}}^{2 i}\right)$ is simple above $p \nmid 2 i$ iff there are $P_{2 i}$-names $\underset{\sim}{I}(n<\omega)$ such that

$$
\begin{aligned}
p \uparrow 2 i \Vdash_{P_{2 i}} " & (\forall n<\omega)\left[I_{n} \subseteq R_{(p \nmid 2 i)\lceil E V E N}\right. \text { countable predense \& } \\
& \left(r \in \underset{\sim}{I_{n}} \Longrightarrow r\right. \text { determines } \\
& p(2 i) \text { to degree } n)] "
\end{aligned}
$$

(3) For $p \in P_{\alpha}$ we define

$$
R_{p} \stackrel{\text { def }}{=}\left\{q \in P_{\alpha}^{\prime}: q \geq \text { apr } p\right\}
$$

with the order inherited from $P_{\alpha}^{\prime}$.

\footnotetext{
${ }^{2}$ Later we shall prove that $P_{\alpha}^{\prime}$ is a dense subset of $P_{\alpha}$, for all $\alpha \leq \omega_{2}$.
} 
(4) If $2 i<\alpha$ and $\tau=\left(t^{\tau}, \mathcal{T}^{\tau}\right)$ is a $P_{2 i}$-name for a condition in $U M$, while $q \in P_{2 i}$, we say that $q$ determines $\tau$ to degree $n$ iff

(i) $q$ forces in $P_{2 i}$ a value to $\mathcal{T}_{\sim}^{\tau} \cap \leq n 2$

(ii) $q$ forces in $P_{2 i}$ a value to $t_{\sim}^{\tau}$,

(iii) for all $\eta \in \leq n 2$, there is $\nu \triangleright \eta$ such that

$$
q \Vdash_{P_{2 i}} " \eta \in \mathcal{T}^{\tau}{ }^{\tau} \Longrightarrow \nu \notin \mathcal{\sim}^{\mathcal{T}^{\tau}} ",
$$

(iv) For all $\eta \in \leq n 2$, there are $\eta_{1} \neq \eta_{2} \triangleright \eta$ such that

$$
q \Vdash_{P_{2 i}} " \eta \in{\underset{\sim}{\mathcal{T}}}^{\tau} \Longrightarrow \eta_{1}, \eta_{2} \in \mathcal{T}^{\mathcal{T}}{ }^{\tau} " .
$$

Claim 4.7. (1)If $p \in P_{\alpha}^{\prime}$ and $\beta<\alpha$, then $p\left\lceil\beta \in P_{\beta}^{\prime}\right.$.

(2) If $p \in P_{\alpha}^{\prime}$, then $p\left\lceil E V E N \in P_{\alpha}^{\prime}\right.$.

Proof of the Claim. This is easily checked, noting that the definition of $p(2 i)$ being simple above $p\lceil 2 i$ only depends on $p\lceil 2 i$, for $2 i \leq \alpha$. $\star$..

Notation 4.8. (1) $p \geq_{\text {apr }}\left(\geq_{\mathrm{pr}}, \geq\right) q$ iff $q \leq_{\mathrm{apr}}\left(\leq_{\mathrm{pr}}, \leq\right) p$.

(2) Let $\bar{Q}=\left\langle P_{\alpha}, Q_{\beta}: \alpha \leq \omega_{2}, \beta<\omega_{2}\right\rangle$ and $\bar{Q}^{\prime}=\left\langle P_{\alpha}^{\prime}: \alpha \leq \omega_{2}\right\rangle$.

(3) $P \stackrel{\text { def }}{=} P_{\omega_{2}}^{\prime}$.

(4) $\chi$ is a fixed large enough regular cardinal, and $<_{\chi}^{*}$ is a fixed well-ordering of $H(\chi)$.

(5) EVEN stands for the set of even ordinals, and $O D D$ for the set of odd ones.

(6) Quantifier $\forall^{*}$ means "for all but finitely many".

Definition 4.9. Suppose that $2 i<\omega_{2}$ and $p \in P_{2 i}$, and $p(2 i)$ is simple above $p \nmid 2 i$, while $\bar{\sim}=\left\langle I_{n}: n\langle\omega\rangle\right.$ are as in Definition 4.6(2). We say that $\bar{I}$ exemplifies the simplicity of $p(2 i)$ above $p \nmid 2 i$. 
Note 4.10. (1) Suppose that $\alpha \leq \omega_{2}$ and $\left\langle\alpha_{n}: n<\omega\right\rangle$ is an increasing sequence of ordinals with $\sup _{n<\omega} \alpha_{n}=\alpha$. Further suppose that $\left\langle q_{n}: n<\omega\right\rangle$ is a sequence such that

(i) $q_{n} \in P_{\alpha_{n}}\left[P_{\alpha_{n}}^{\prime}\right]$ for all $n$,

(ii) $q_{n+1} \uparrow \alpha_{n}=q_{n}$.

Then $q \stackrel{\text { def }}{=} \bigcup_{n<\omega} q_{n}$ is a condition in $P_{\alpha}\left[P_{\alpha}^{\prime}\right]$.

(2) For every $\alpha<\omega_{2}$ we have $\Vdash_{P_{\alpha}}$ " $Q_{\alpha}$ is proper."

(3) If $\alpha \leq \omega_{2}$ and $p, p^{\prime}$ are such that $p \leq_{\text {apr }} p^{\prime} \in P_{\alpha}^{\prime}$, then

$$
R_{p^{\prime}}=\left\{q \in R_{p}: q \geq p^{\prime}\right\}
$$

(4) If $p \leq_{\text {apr }} p^{\prime} \in P^{\prime}$, then $\delta^{*}(p)=\delta^{*}\left(p^{\prime}\right)$.

Notation 4.11. (1) Given $\gamma<\omega_{2}$ even. We let ${\underset{\sim}{\gamma}}_{\gamma}$ be a $P_{\gamma}$-name for the dominating real added by ${\underset{\sim}{\gamma}}_{\gamma}$.

(2) Suppose that $\beta<\alpha \leq \omega_{2}$ and $A \subseteq P_{\alpha}^{\prime}$. We define

$$
A \uparrow \beta \stackrel{\text { def }}{=}\{s \uparrow \beta: s \in A\} .
$$

(3) For $\alpha \leq \omega_{2}$ and $J \subseteq P_{\alpha}^{\prime}$, we say that $J$ is $\leq_{\text {pr}^{-}}$open iff

$$
(\forall q \in J)\left(\forall p \geq_{\operatorname{pr}} q\right)(p \in J)
$$

We say that $J$ is $\leq_{\mathrm{pr}^{-}}$dense above $p \in P_{\alpha}^{\prime}$ iff

$$
\left(\forall q \in P_{\alpha}^{\prime}\right)\left[q \geq_{\text {pr }} p \Longrightarrow(\exists r \in J)\left(r \geq_{\text {pr }} q\right) \cdot\right]
$$

Observation 4.12. Suppose that $\beta<\alpha \leq \omega_{2}$ and $p \in P_{\alpha}^{\prime}$. Further suppose that $J \subseteq P_{\alpha}^{\prime}$ is $\leq_{\text {pr-open }}$ and $\leq_{\text {pr }}$-dense above $p$.

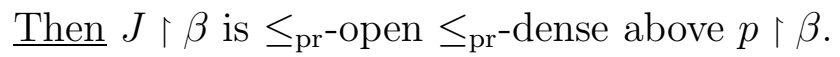


Observation 4.13. Suppose that $\alpha \leq \omega_{2}$ and $p \leq q \in P_{\alpha}$, and let as define $r$ as follows:

$$
r(\beta) \stackrel{\text { def }}{=} \begin{cases}p(\beta) & \text { if } \beta \in(E V E N \cap \operatorname{Dom}(p)) \\ q(\beta) & \text { otherwise }\end{cases}
$$

letting $\operatorname{Dom}(r)=\operatorname{Dom}(q)$. Then $r \in P_{\alpha}$ and $r$ has the following properties:

(i) $p \leq_{\text {pr }} r \leq_{\text {apr }} q$

(ii) $\neg(q \uparrow \alpha \Vdash " r(\alpha)=q(\alpha) ") \Longrightarrow \alpha \in \operatorname{Dom}(p)$.

(iii) If there is $\delta^{*}$ such that for all $\beta \in \operatorname{Dom}(q)$ we have $\Vdash_{P_{\beta}}$ "lt $(q)=\delta^{*}$,

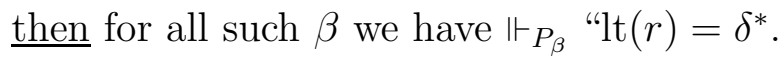

Notation 4.14. Suppose that $\alpha \leq \omega_{2}$ and $p \leq q \in P_{\alpha}$. Then $r$ defined as in Observation 4.13 is denoted by $\operatorname{intr}(p, q)$.

Claim 4.15. Given $\alpha \leq \omega_{2}$ and $p \in P_{\alpha}^{\prime}$. Then

$$
p \Vdash_{P_{\alpha}} \text { " } R_{p} \text { is a } c c c \text { partial order". }
$$

(More is true, see Lemma 6.6.)

Proof of the Claim. By induction on $\alpha$, for all $p \in P_{\alpha}^{\prime}$ simultaneously. There are two eventful cases of the induction.

$\alpha=\beta+1, \beta$ even. Note that $R_{p} \subseteq R_{p \nmid \beta} *\left\{\underset{\sim}{\mathcal{\tau}} \in U U_{\sim} M: \underset{\sim}{\tau} \geq p(\beta)\right\}$ is a dense suborder. (Or see the proof of Claim 5.1 (1) $)_{e}^{\alpha}$ case $\alpha=\beta^{*}+1$ and $\beta^{*}$ even.)

$$
\begin{aligned}
& \underline{\operatorname{cf}(\alpha)=\aleph_{0}} . \text { Given }\left\{r_{i}: i<\omega_{1}\right\} \subseteq R_{p} . \text { For } i<\omega_{1} \text { let } \\
& F_{i} \stackrel{\text { def }}{=}\left\{\beta \in \operatorname{Dom}(p): \neg\left(r_{i} \uparrow \beta \Vdash “ r_{i}(\beta)=p(\beta) "\right)\right\} \text {. }
\end{aligned}
$$

Without loss of generality, $\left\{F_{i}: i<\omega_{1}\right\}$ forms a $\Delta$-system with root $F^{*}$, and now the conclusion follows by the induction hypothesis. $\star 4.15$ 
Claim 4.16. Suppose that $\alpha \leq \omega_{2}$ and $q, r \in P_{\alpha}^{\prime}$ are such that $p \leq_{\text {apr }} r$ and $p \leq_{\text {pr }} q$. Let us define $r+q$ by letting $\operatorname{Dom}(r+q)=\operatorname{Dom}(q)$ and for $\beta \in \operatorname{Dom}(r+q)$

$$
(r+q)(\beta) \stackrel{\text { def }}{=} \begin{cases}r(\beta) & \text { if } \beta E V E N \in \operatorname{Dom}(r) \\ q(\beta) & \text { otherwise. }\end{cases}
$$

Then $r+q \in R_{q}$ and $r+q \geq_{\mathrm{pr}} r$.

Proof of the Claim. The proof is by induction on $\alpha$, for all conditions in $P_{\alpha}^{\prime}$ simultaneously. The eventful case of the induction is $\alpha=\beta+1, \beta$ even.

We need to prove that $(r+q)(\beta)$ is simple above $(r+q) \vdash \beta$.

Case 1. $\beta \in \operatorname{Dom}(r)$.

Let $\left\langle I_{n}: n<\omega\right\rangle$ exemplify that $r(\beta)$ is simple above $r\lceil\beta$. For $n<\omega$ let

$$
J_{n} \stackrel{\text { def }}{=}\left\{s+[(r+q) \uparrow \beta] \uparrow E V E N: s \in I_{n}\right\} .
$$

By the induction hypothesis we have that $(r+q)\left\lceil\beta\right.$ forces $J_{n}$ to be a countable subset of $R_{[(r+q)\lceil\beta]\lceil E V E N}$. We finish by noticing that it is also forced by $(r+q) \uparrow \beta$ that $J_{n}$ is predense in $R_{[(r+q)\lceil\beta]\lceil E V E N}$.

Case $2 . \beta \notin \operatorname{Dom}(r)$.

Let now $\left\langle I_{n}: n<\omega\right\rangle$ exemplify that $q(\beta)$ is simple above $q \uparrow \beta$. Let for $n<\omega$

$$
\underset{\sim}{K_{n}} \stackrel{\text { def }}{=}\left\{z \in R_{[(r+q) \mid \beta] \mid E V E N}:\left(\exists s \in I_{n}\right)[z \geq s]\right\},
$$

and let $J_{n}$ be countable predense $\subseteq \underset{\sim}{K_{n}}$. It is easily seen that $\left\langle J_{n}: n<\omega\right\rangle$ exemplify that $(r+q)(\beta)$ is simple above $(r+q) \uparrow \beta$.

$\star 4.16$

Note 4.17. In the notation of Claim 4.16, $r+q=(r\lceil E V E N)+q$.

Notation 4.18. Suppose that $p$ and $q$ are as in Claim 4.16, and $R \subseteq R_{p}$. Then $R+q \stackrel{\text { def }}{=}\{r+q: r \in R\}$. 


\section{Properness}

Claim 5.1. Given $\alpha \leq \omega_{2}$. The following holds.

$(0)^{\alpha}$ Suppose $2 i<\alpha$ and $q \in P_{2 i}^{\prime}$ determines $\underset{\sim}{\tau}=\left(t^{\tau}, \mathcal{T}^{\tau}\right)$ to degree $n$. Then $q\lceil E V E N$ determines $\tau$ to degree $n$.

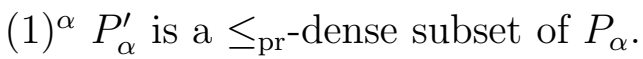

$(2)^{\alpha}$ Suppose that $N \prec(H(\chi), \in)$ countable, $\left\{p, \alpha, \bar{Q}, \bar{Q}^{\prime}\right\} \subseteq N$, where $p$ is some element of $P_{\alpha}^{\prime}$. Further assume that $J \in N$ is forced by $p$ to be a $\leq_{\text {pr}}$-open $\leq_{\text {pr }}$-dense above $p$ subset of $\left\{q \in P_{\alpha}^{\prime}: p \leq_{\text {pr }} q\right\}$, and $u$ is finite $\subseteq O D D \cap \operatorname{Dom}(p)$, while $\epsilon>0$. In addition, suppose that for $\gamma \in u$ we have a $P_{\gamma}$-name $\mathcal{\sim}_{\gamma}$ (not necessarily in $\left.N\right)$ such that $p\left\lceil\gamma \Vdash{ }^{~ " \tau_{\gamma}}\right.$ is finite $\subseteq \underset{\sim}{T_{N \cap \omega_{1}}^{\gamma}}$ ". Let $\underset{\sim}{\overline{\mathcal{I}}} \stackrel{\text { def }}{=}\left\langle\mathcal{\tau}_{\gamma}: \gamma \in u\right\rangle$.

Then there is $q \in P_{\alpha}^{\prime}$ such that

\begin{tabular}{|c|c|}
\hline$(*)_{p, q, N, J, u, \epsilon, \bar{\tau}}^{\alpha}$ meaning & 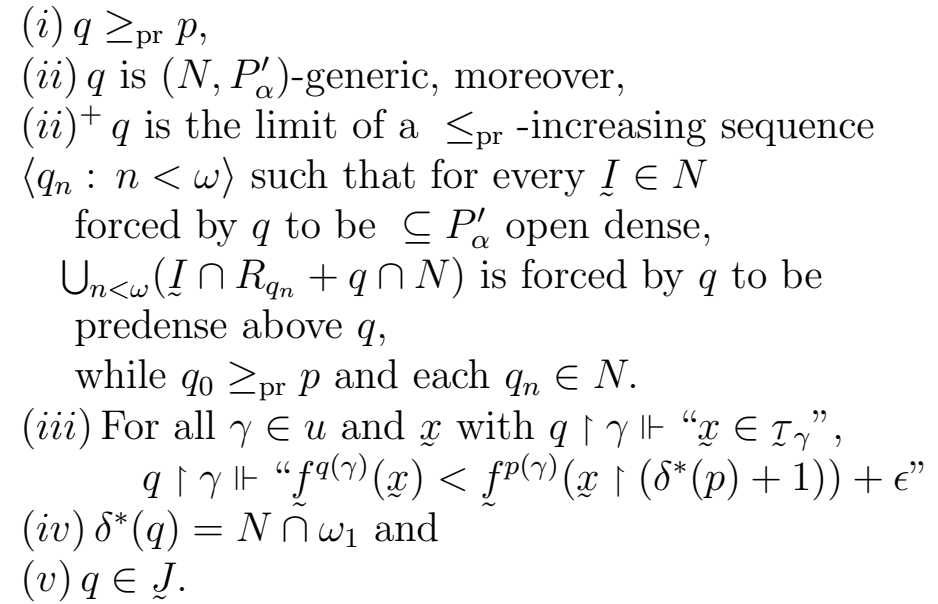 \\
\hline
\end{tabular}

Notation 5.2. Suppose that $(*)_{p, q, N, J, u, \epsilon, \bar{\tau}}^{\alpha}$ holds for some appropriate values of $\alpha, p, q, N, J, u, \epsilon, \underset{\sim}{\mathcal{\tau}}$, and that $\left\langle q_{n}: n<\omega\right\rangle$ is a sequence as in the definition of $(*)_{p, q, N, J, u, \epsilon, \tau_{\sim}^{*}}^{\alpha} \quad$ We say that $\left\langle q_{n}: n<\omega\right\rangle$ exemplifies that $(*)_{p, q, N, J, u, \epsilon, \bar{\tau}}^{\alpha}$ holds.

Proof of the Claim. The proof is by induction on $\alpha$, proving $(0)^{\alpha},(1)^{\alpha}$ and $(2)^{\alpha}$ simultaneously. However, we shall formulate four additional statements to help us carry the induction. These statements are denoted by $(1)_{e}^{\alpha},(1)^{+, \alpha}$, 
$(1)_{o}^{\alpha}$ and $(2)^{+, \alpha}$. We shall prove by induction on $\alpha$ that $(0)^{\alpha},(1)_{e}^{\alpha},(1)^{+, \alpha}$, $(1)_{o}^{\alpha}$, and $(2)^{+, \alpha}$ hold. As $(2)^{+, \alpha}$ is clearly a strengthening of $(2)^{\alpha}$ and $(1)^{+, \alpha}$ of $(1)^{\alpha}$, this suffices.

Description of $(1)_{e}^{\alpha},(1)^{+, \alpha},(1)_{o}^{\alpha}$ and $(2)^{+, \alpha}$.

(1) $)_{e}^{\alpha}$ Assume that $\alpha=\beta+1$ and $\beta$ is even, while $p \in P_{\alpha}$ is such that $p \nmid \beta \in P_{\beta}^{\prime}$, and $p$ forces $J \subseteq P_{\alpha}^{\prime}$ to be $\leq_{\mathrm{pr}^{-}}$-open and $\leq_{\mathrm{pr}}$-dense above $p$. Further assume that $N \prec(H(\chi), \in)$ is countable and $\left\{p, \beta, \bar{Q}, \bar{Q}^{\prime}, J\right\} \subseteq N$. Suppose $(*)_{p \nmid \beta, r, N, J \mid \beta, u, \epsilon, \bar{\tau}}^{\beta}$ for some appropriate $u, \bar{\tau}$ and $\epsilon$.

Then $q \stackrel{\text { def }}{=} r \cup\{(\beta, p(\beta))\} \in P_{\alpha}^{\prime}$ and $q \geq_{\text {pr }} p$. If $p \in P_{\alpha}^{\prime}$, then $(*)_{p, q, N, J, u, \in, \bar{\tau}}^{\alpha}$.

$(1)^{+, \alpha}$ Suppose that $p \in P_{\alpha}, \beta \leq \alpha$ and $r \in P_{\beta}^{\prime}$ are such that for some $p^{\prime} \in P_{\beta}^{\prime}$ with $p^{\prime} \geq_{\mathrm{pr}} p\lceil\beta$ and some appropriate $N, J, \epsilon, \bar{\tau}$ we have $(*)_{p^{\prime}, r, N, J, u, \epsilon, \bar{\tau}}^{\beta}$. Then there is $q \in P_{\alpha}^{\prime}$ such that $q \uparrow \beta=r$ and $q \geq_{\text {pr }} p$.

$(1)_{o}^{\alpha}$ Suppose that $\alpha=\beta+1$ and $\beta$ is odd. Given $N \prec(H(\chi), \in)$ countable such that $\alpha, \bar{Q}, \bar{Q}^{\prime} \in N$ and let $J, u, \epsilon$ and $\bar{\tau}$ be as in the assumptions of $(2)^{\alpha}$. Let $\delta \stackrel{\text { def }}{=} N \cap \omega_{1}$. Let $\left\{I_{n}: n<\omega\right\}$ enumerate all $P_{\beta}$-names of open dense subsets of $Q_{\beta}$ which are elements of $N$. Further assume that $\left\langle r_{n}: n<\omega\right\rangle$ exemplifies that $(*)_{p \nmid \beta, r, N, J\lceil\beta, u \cap \beta, \epsilon, \bar{\tau} \uparrow \beta}^{\beta}$ holds.

Now assume that $\left\langle p_{n}: n<\omega\right\rangle$ is a sequence of conditions in $P_{\alpha}^{\prime}$ with the following properties:

(a) $p_{n}\left\lceil\beta=r_{n}\right.$ and $p_{n} \in N$.

(b) $p_{n} \leq_{\text {pr }} p_{n+1}$.

(c) There is a series $\Sigma_{n<\omega} \epsilon_{n}$ with $\Sigma_{n<\omega} \epsilon_{n}<\epsilon$, such that for each $n<\omega$ the following statement $\oplus$ is forced by $P_{\beta}$ :

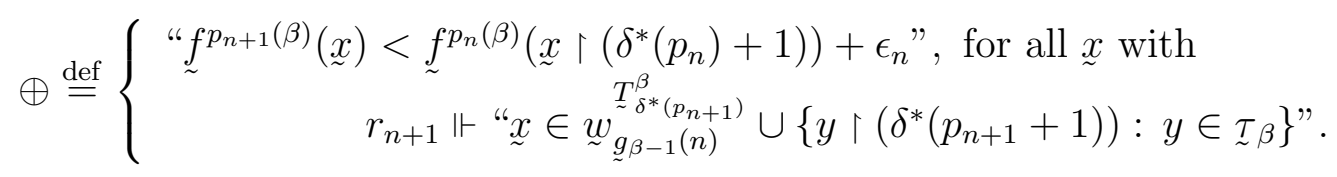

(e) $r \Vdash_{P_{\beta}}$ " $p_{n+1}(\beta) \in \underset{\sim}{I}$ ". 
Then the following defines a condition $q$ in $P_{\alpha}^{\prime}$ :

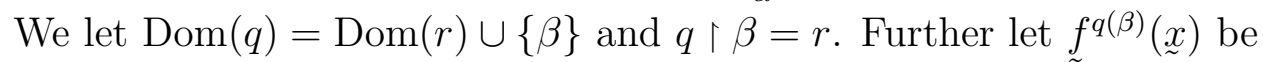

$$
{\underset{\sim}{f}}^{p_{n}(\beta)}(\underset{\sim}{x})
$$

if for some $\underset{\sim}{j}$ with $r_{n} \Vdash " \underset{\sim}{j} \in C_{\sim}^{p_{n}(\beta)}$, we have

$$
r_{n} \Vdash “ \underset{\sim}{x} \in \underset{\sim}{\underset{g_{\beta}}{T_{j}^{\beta}}} \underset{\sim_{j-1}(n)}{\sim^{\prime}} \cup\left\{y \uparrow\left(\delta^{*}\left(p_{n}\right)+1\right): y \in \mathcal{I}_{\beta}\right\} "
$$

and let it be

$$
\Sigma_{n<\omega} f_{\sim}^{p_{n}(\beta)}\left(\underset{\sim}{x} \uparrow\left(\delta^{*}\left(p_{n}\right)+1\right)\right)
$$

if $r \Vdash " x \in \underset{\sim}{T_{\delta}^{\beta}}$ ".

We let $C_{\sim}^{q(\beta)} \stackrel{\text { def }}{=}\left(\cup_{n<\omega} C^{p_{n}(\beta)}\right) \cup\{\delta\}$. Let $\Psi^{q(\beta)} \stackrel{\text { def }}{=} \bigcup_{n<\omega} \Psi^{p_{n}(\beta)}$.

Moreover,

$$
(*)_{p, q, N, u \cup\{\beta\}, J, \epsilon, \bar{\tau}}^{\alpha} \text {. }
$$

$(2)^{+, \alpha}$ For every $\beta \leq \alpha$ and $p, N, J, u, \epsilon, \bar{\tau}$ as in the hypothesis of $(2)^{\alpha}$, and $r \in P_{\beta}^{\prime}$ such that $(*)_{p\lceil\beta, r, N, J\lceil\beta, u \cap \beta, \epsilon, \overline{\mathcal{T}}\lceil\beta}^{\beta}$, there is $q \in P_{\alpha}^{\prime}$ such that $(*)_{p, q, N, J, u, \epsilon, \bar{\tau}}^{\alpha}$ and $q \uparrow \beta=r$.

Proof of $(0)^{\alpha},(1)_{e}^{\alpha},(1)^{+, \alpha},(1)_{o}^{\alpha}$, and $(2)^{+, \alpha}$.

$\underline{\alpha=0}$. Trivial.

$\alpha=\beta^{*}+1$ and $\beta^{*}$ is even.

$(0)^{\alpha}$ Without loss of generality, $2 i=\beta^{*}$. Suppose that the claim is not true. Applying $(1)^{\beta^{*}}$, there are $q_{1}, q_{2} \geq_{\text {pr }} q\left\lceil E V E N\right.$ both in $P_{\beta^{*}}^{\prime}$, and $q_{1}, q_{2}$ force contradictory statements about $\tau$ to degree $n$, while determining it. But then $q_{1}+q$ and $q_{2}+q$ both extend $q$ and force contradictory information on a fact which $q$ already determines.

$(1)_{e}^{\alpha}$ Hence $\beta=\beta^{*}$. It is easily seen that $q \in P_{\alpha}$ and $q \geq_{\text {pr }} p$. By the choice of $r$, in order to see that $q \in P_{\alpha}^{\prime}$ we only need to check that $p(\beta)$ is simple above $q \uparrow \beta$. Given $n<\omega$. Let

$$
\stackrel{\text { def }}{=}\left\{s \in P_{\beta}: s \text { determines } p(\beta) \text { to degree } n\right\} .
$$

Hence $I \subseteq P_{\beta}$ is forced by $p\lceil\beta$ to be open dense above $p\lceil\beta$, and certainly $I \in N$. Let $I_{\sim}^{\prime} \stackrel{\text { def }}{=} \underset{\sim}{I} \cap P_{\beta}^{\prime}$. By the induction hypothesis $(1)^{\beta}$, it is forced by 
$p\left\lceil\beta\right.$ that $\underset{\sim}{I^{\prime}}$ is an open dense subset of $P_{\beta}^{\prime}$. So, by the choice of $r$ as a limit of a purely increasing sequence $\left\langle r_{m}: m<\omega\right\rangle$, (see $\left.(i i)^{+}\right)$we have that

$$
\underset{\sim}{I_{n}} \stackrel{\text { def }}{=} \bigcup_{m<\omega}\left(\underset{\sim}{I^{\prime}} \cap\left(R_{r_{m}}+r\right) \uparrow E V E N \cap N\right)
$$

is forced by $r \uparrow E V E N$ to be predense above $r \uparrow E V E N$. Certainly it is also forced by $r$ that $I_{n}$ is countable and consists of conditions which are in $R_{(q\lceil\beta)\lceil E V E N}$, so $\underset{\sim}{I_{n}}$ is as required. This shows that $q \in P_{\alpha}^{\prime}$.

Suppose that $p \in P_{\alpha}^{\prime}$. As we have $\Vdash_{P_{\beta}}$ " $Q_{\beta}$ is $c c c$ ", it follows by the usual arguments that $q$ is $\left(N, P_{\alpha}\right)$-generic. As we have just proved that $P_{\alpha}^{\prime}$ is $\leq_{\text {pr-dense }} \subseteq P_{\alpha}$, by the choice of $\left\langle r_{n}: n<\omega\right\rangle$ we can find a subsequence $\left\langle r_{n_{k}}: k<\omega\right\rangle$ such that choosing $q_{k} \stackrel{\text { def }}{=} r_{n_{k}} \cup\{(\beta, p(\beta))\}$ we'll have shown that $(i i)^{+}$from the definition of $(*)_{p, q, N, J, u, \epsilon, \bar{\tau}}^{\alpha}$ holds. If $u \subseteq O D D \cap \alpha$, then in fact $u \subseteq \beta$, so (iii) holds as well, by the choice of $r$. It is also easily seen that $(i v)$ and $(v)$ hold, noticing that $\underset{\sim}{J} \beta \beta$ is $\leq_{\mathrm{pr}}$-dense and $\leq_{\mathrm{pr}}$-open above $p \uparrow \beta$.

$(1)^{+, \alpha}$ Given $p \in P_{\alpha}$. Without loss of generality, $\beta=\beta^{*}$. Now apply $(1)_{e}^{\alpha}$ to $r \cup\left\{\left(\beta^{*}, p\left(\beta^{*}\right)\right)\right\}$.

$(1)_{o}^{\alpha}$ Follows by the induction hypothesis, as $\beta$ is even.

$(2)^{+, \alpha}$ Without loss of generality, $\beta=\beta^{*}$. We let $q \stackrel{\text { def }}{=} r \cup\{(\beta, p(\beta))\}$. By $(1)_{e}^{\alpha}$ it follows that $(*)_{p, q, N, J, u, \epsilon, \bar{\tau}}^{\alpha}$ holds.

$\alpha=\beta^{*}+1$ and $\beta^{*}$ is odd.

$(1)_{e}^{\alpha}$ Does not apply.

$(1)^{+, \alpha}$ Follows by the induction hypothesis $(1)^{+, \beta^{*}}$.

$(1)_{o}^{\alpha}$ Hence $\beta^{*}=\beta$. This is like the proof of Claim 2.8, but we also get to use Claim 2.11. We first show that $\vdash_{P_{\beta}} " q(\beta) \in \underset{Q_{\beta}}{ }$. . It is easily seen that

$$
\Vdash_{P_{\beta}} \text { " } C^{q(\beta)} \text { is a closed subset of } \delta+1 \text { with the last element } \delta " \text {. }
$$

It is also easy to see, by the choice of $\left\langle p_{n}: n<\omega\right\rangle$, that $P_{\beta}$ forces that $\Psi^{q(\beta)}$ is a countable set of promises, and that $\underset{\sim}{\Gamma} \in \underset{\sim}{\Psi} \Psi^{q(\beta)} \Longrightarrow \delta \in \underset{\sim}{C}(\underset{\sim}{\Gamma})$ (because the promises are in $N)$, and $\left.\underset{\sim}{C} \underset{\sim}{\Gamma}) \supseteq{\underset{\sim}{C}}^{q(\beta)} \backslash \min (\underset{\sim}{C} \underset{\sim}{\Gamma})\right)$. We have to check that $P_{\beta}$ forces $f_{\sim}^{q(\beta)}$ to be a well defined function. 
All information in the next 3 paragraphs is either true or forced by $r$ to be true, and which one is the case is clear from the context:

For $\underset{\sim}{x} \in \operatorname{Dom}\left({\underset{\sim}{f}}^{q(\beta)}\right)$ for which ${\underset{\sim}{f}}^{q(\beta)}(\underset{\sim}{x})$ is defined by the first clause of its definition, the fact that $f_{\sim}^{q(\beta)}(\underset{\sim}{x})$ is well defined, follows from the fact that $p_{n}$ are increasing. For those $\underset{\sim}{x} \in \operatorname{Dom}\left({\underset{\sim}{f}}^{q(\beta)}\right)$ for which ${\underset{\sim}{f}}^{q(\beta)}(\underset{\sim}{x})$ is defined by the second clause of the definition, we have $r \Vdash \sim_{\sim}^{x} \in \underset{\sim}{\tilde{T}_{\delta}^{\beta}}$ ". Hence $\underset{\sim}{x}$ is a $P_{\beta-1}$-name (this is where we use the fact that $\underset{\sim}{T^{\beta}}$ is a $P_{\beta-1}$-name.) We define a $P_{\beta-1}$-name $\underset{\sim}{h}$ of a function from $\omega$ to $\omega$ by $\underset{\sim}{h}(n)=m$ iff $\underset{\sim}{x} \uparrow\left(\delta^{*}\left(p_{n}\right)+1\right)$ is the $m$-th element of the increasing enumeration of $\underset{\sim}{T_{\delta^{*}}^{\beta}\left(p_{n}\right)}$.

By the definition of $g_{\beta-1}$ we have that for all but finitely many $n$, it is forced by $P_{\beta}$ that $\underset{\sim}{h}(n)<{\underset{\sim}{\beta}-1}_{\beta-1}(n)$. Hence for all but finitely many $n$ we have that $\oplus$ from $(\mathrm{c})$ in $(1)_{o}^{\alpha}$ holds for $\underset{\sim}{x}$ in question. Hence $\underset{\sim}{f}{ }^{q(\beta)}(\underset{\sim}{x})$ is well defined.

Now it is also obvious that $f^{q(\beta)}$ is forced to be a partial monotonically increasing function into $\mathbb{Q}$. We can also see that the domain of $f^{q(\beta)}$ is forced to be $\bigcup_{i \in C^{q(\beta)}} \underset{\sim}{T}{ }_{i}^{\beta}$, as this follows by the fact that $\Vdash_{P_{\beta-1}}$ " $g_{\beta-1}$ diverges to $\infty$."

The rest is easy to check.

$(2)^{+, \alpha}$ By the induction hypothesis, without loss of generality we have $\beta^{*}=\beta$ and $u=\left\{\beta^{*}\right\}$. For $n<\omega$ let $\epsilon_{n} \stackrel{\text { def }}{=} \epsilon / 2^{n+2}$.

Let $\delta \stackrel{\text { def }}{=} N \cap \omega_{1}$. By Fact 2.7, we can find a sequence $\left\langle p_{n}: n<\omega\right\rangle$ which satisfies (a)-(e) in the statement of $(1)_{o}^{\alpha}$, where we have chosen $\left\langle r_{n}: n<\omega\right\rangle$ to exemplify $(*)_{p \nmid \beta, r, N, J\lceil\beta, u \cap \beta, \epsilon, \bar{\tau}\lceil\beta}^{\beta}$.

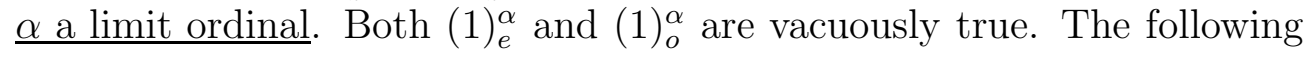
proof proves both $(1)^{+, \alpha}$ and and $(2)^{+, \alpha}$. Given $p^{-} \in P_{\alpha}$ and $\beta \leq \alpha$.

Case 1. $\operatorname{cf}(\alpha)=\aleph_{0}$.

Let $\left\langle\alpha_{n}: n<\omega\right\rangle$ be a sequence in $N$ which is increasing and cofinal in $\alpha$, with $\alpha_{0}=\beta$. Let $p \stackrel{\text { def }}{=} p_{0} \geq_{\operatorname{pr}} p^{-} \uparrow \beta$ be such that $p_{0} \in P_{\beta}^{\prime}$. Without loss of generality, $p_{0} \in N$. Let $\left\langle u_{n}: n<\omega\right\rangle$ be an increasing sequence of finite subsets of $O D D \cap N \cap \omega_{2}$, with $\bigcup_{n<\omega} u_{n}=N \cap O D D \cap \omega_{2}$. Let $\delta \stackrel{\text { def }}{=} N \cap \omega_{1}$. Let $\left\langle\delta_{n}: n<\omega\right\rangle$ be an increasing sequence of ordinals, cofinal in $\delta$, and such that $\delta_{0}=\delta^{*}(p)$. We are assuming that the assumptions of $(2)^{+, \alpha}$ hold.

By induction on $n<\omega$ we shall construct two sequences $\left\langle q_{n}: n<\omega\right\rangle$ and $\left\langle p_{n}: n<\omega\right\rangle$ such that 
(A) $p_{0}=p$ and $q_{0}=r$.

(B) $p_{n} \in P_{\alpha}^{\prime} \cap N$ and $q_{n} \in P_{\alpha_{n}}^{\prime}$.

(C) $\delta^{*}\left(p_{n}\right) \geq \delta_{n}$.

(D) $(*)_{p_{n}, q_{n}, N, J\left\lceil\alpha_{n}, u_{n} \cap \alpha_{n}, \epsilon, \bar{\tau}\left\lceil\alpha_{n}\right.\right.}^{\alpha_{n}}$.

(E) $p_{n+1} \geq_{\text {pr }} p_{n}$.

(F) $q_{n+1} \uparrow \alpha_{n}=q_{n}$ and $p_{n+1} \geq_{\operatorname{pr}} p^{-} \uparrow \alpha_{n+1}$ and $p_{n+1} \uparrow \alpha_{n}=q_{n}$.

The induction goes through without problems. We now take $q=\bigcup_{n<\omega} q_{n}$.

Case 2. $\operatorname{cf}(\alpha)=\aleph_{1}$.

The conclusion follows by the induction hypothesis.

$\star 5.1$

Remark 5.3. Claim 5.1 in particular implies that $P$ is a proper forcing notion.

Claim 5.4. (1) For all $\alpha<\omega_{2}$ we have

(i) $\emptyset \Vdash_{P_{\alpha}} "\left|Q_{\alpha}\right|$ has $\aleph_{2}$-pic"

(ii) $\emptyset \Vdash_{P_{\alpha}} " 2^{\aleph_{0}}=\aleph_{1} "$.

(iii) $P_{\alpha}^{\prime \prime} \stackrel{\text { def }}{=}\left\{p \in P_{\alpha}^{\prime}:(\forall i \in \operatorname{Dom}(p))\left[p(i)\right.\right.$ is a name from $H_{<\aleph_{1}}($ Ord $\left.\left.)\right]\right\}$ is dense in $P_{\alpha}^{\prime}$.

(2) $P$ has $\aleph_{2}-c c$.

Proof of the Claim. The proof uses Fact 2.6 and is like the corresponding proof for countable support iterations, [Sh -f VIII, §2], which we quoted as Fact 2.5. Of course, notice that ccc trivially implies $\aleph_{2}$-pic** $\star 5.4$

Lemma 5.5. It is possible to arrange the bookkeeping so that $V^{P} \models$ "there are no Suslin trees (in fact, all Aronszajn trees are special)."

Proof of the Lemma. This is standard, by $V \models " 2^{\aleph_{1}}=\aleph_{2}$ " and Fact 2.6. $\star 5.5$ 


\section{Sweetness revisited}

Notation 6.1. Suppose that $\alpha \leq \beta \leq \omega_{2}$ and $p, q \in P_{\beta}^{\prime}$. We say

(0) Suppose $p \leq q$. We write $p(\alpha) \neq q(\alpha)$ iff $\neg(q\lceil\alpha \Vdash " q(\alpha)=p(\alpha)$ ").

(1) $p \leq^{+} q$ iff $p \leq q$ and for all $\alpha$ even with $p(\alpha) \neq q(\alpha)$, we have that ${\underset{\sim}{t}}^{q(\alpha)}$ is an object $t^{q(\alpha)}$, not just a name.

(2) $p \leq_{\text {apr }}^{+} q$ iff $\left[p \leq_{\text {apr }} q\right.$ and $\left.p \leq^{+} q\right]$.

$(3) \geq^{+}$and $\geq_{\text {apr }}^{+}$are defined in the obvious manner.

(4) $R_{p}^{+} \stackrel{\text { def }}{=}\left\{r \in R_{p}: r \geq_{\text {apr }}^{+} p\right\}$.

Each $R_{p}^{+}$will be a sweetness model.

Claim 6.2. Suppose that $\alpha \leq \omega_{2}$ and $p \leq_{\text {apr }} q \in P_{\alpha}^{\prime}$. Then for some $q^{+} \in P_{\alpha}^{\prime}$ we have $p, q \leq_{\text {apr }}^{+} q^{+}$.

Proof of the Claim. By induction on $\alpha$. The only eventful case of the induction is the case when $\alpha=\beta+1$ for some $\beta$ even. As $p(\beta)$ is simple above $p \uparrow \beta$ and $q \uparrow \beta \in R_{p \uparrow \beta}$, we can find $z \in R_{p \uparrow \beta}$ with $z \geq$ apr $q$ and such that $z$ decides the value of ${\underset{\sim}{t}}^{p(\beta)}$. By the induction hypothesis we find $z^{+} \in R_{z}^{+}$such that $z^{+} \geq_{\text {apr }} p \uparrow \beta, q \uparrow \beta, z$. We define $t$ by letting

$$
t \stackrel{\text { def }}{=}\left\{\eta: z^{+} \Vdash " \eta \in{\underset{\sim}{t}}^{p(\beta)} "\right\},
$$

and let $q^{+} \stackrel{\text { def }}{=} z^{+} \cup\left\{\left(\beta,\left(t,{\underset{\sim}{\mathcal{T}}}^{p(\beta)}\right)\right)\right\} . \star 6.2$

Definition 6.3. Suppose that $\hat{\alpha} \leq \omega_{2}$ and $p \in P_{\hat{\alpha}}^{\prime}$ with $\operatorname{Dom}(p) \subseteq E V E N$. We define:

(1) For $r \in R_{p}$ we let $\operatorname{Dom}_{p}^{*}(r) \stackrel{\text { def }}{=}\{\beta \in \operatorname{Dom}(p): r(\beta) \neq p(\beta)\}$.

$\operatorname{Dom}_{p}^{*}(r)$ is the domain of $r$ in $R_{p}$. 
(2) A sequence $\bar{x}$ is called an assignment for $p$ if for some $\alpha>\sup (\operatorname{Dom}(p))$, which we denote by $\alpha(\bar{x})$, we have

$$
\bar{x}=\left\langle\left\langle A_{m}^{\gamma}: m<\omega\right\rangle: \gamma \in \operatorname{Dom}(p) \cup\{\alpha\}\right\rangle
$$

and each $A_{m}^{\gamma}$ is a directed subset of $R_{p \nmid \gamma}^{+}$, while $\bigcup_{m<\omega} A_{m}^{\gamma}$ is dense in $R_{p \nmid \gamma}^{+}$; or if $\bar{x}$ has an initial segment with domain $\left(\operatorname{Dom}(p) \cup\left\{\alpha^{\prime}\right\}\right)$ which has the just mentioned properties.

We use the notation ${ }^{\bar{x}} A_{m}^{\gamma}$ to denote $\bar{x}(\gamma, m)$.

The intended meaning of an assignment is an enumeration of equivalence classes of $R_{p\lceil\gamma}^{+}$for $\gamma$ in $\operatorname{Dom}(\bar{x})$.

(3) For $a \in\left[\omega_{2}\right]^{\leq \aleph_{0}}$, we define

$$
\begin{aligned}
& \mathrm{FA}_{a} \stackrel{\text { def }}{=}\left\{\left\langle\left(\beta_{j}, t_{j}\right): j<j^{*}\right\rangle: \quad j^{*}<\omega \& \beta_{j} \in a \cap E V E N \& \beta_{j}\right. \text { are } \\
&\text { increasing \& } \left.t_{j} \text { is a finite subtree of }<_{2}\right\} .
\end{aligned}
$$

The intended meaning of $\mathrm{FA}_{a}$ is to be a formal $E_{0}$-equivalence class.

(4) For $y \in \mathrm{FA}_{\operatorname{Dom}(p)}$, we let

$$
\begin{aligned}
A_{p}^{y} \stackrel{\text { def }}{=}\left\{r \in R_{p}^{+}:\right. & \operatorname{Dom}_{p}^{*}(r)=\{\beta:(\exists t)((\beta, t) \in \operatorname{Rang}(y))\} \& \\
& \left.(\beta, t) \in \operatorname{Rang}(y) \Longrightarrow t^{r(\beta)}=t\right\} .
\end{aligned}
$$

(5) $\bar{y}$ is a formal 0-canonical assignment for $p$ if for some $\alpha>\sup (\operatorname{Dom}(p))$, which we denote by $\alpha(\bar{y})$, we have

$$
\bar{y}=\left\langle\bar{y}^{\gamma}=\left\langle y_{m}^{\gamma}: m<\omega\right\rangle: \gamma \in \operatorname{Dom}(p) \cup\{\alpha\}\right\rangle
$$

and $\left\{y_{m}^{\gamma}: m<\omega\right\}$ is a list, possibly with repetitions, of $\mathrm{FA}_{\operatorname{Dom}(p\lceil\gamma)}$, for $\gamma \in \operatorname{Dom}(p) \cup\{\alpha(\bar{y})\}$; or if $\bar{y}$ has an initial segment of domain $\left(\operatorname{Dom}(p) \cup\left\{\alpha^{\prime}\right\}\right)$ which has the just mentioned properties.

A formal 0-canonical assignment gives a list of formal $E_{0}$-equivalence classes. The main definition of this section, Definition 6.5, will deal with formal $E_{n}$-equivalence classes.

(6) An assignment $\bar{x}$ is a 0-canonical assignment for $p$ if for some formal 0canonical assignment $\bar{y}$ for $p$, we have ${ }^{\bar{x}} A_{m}^{\gamma} \subseteq A_{p}^{y_{m}^{\gamma}}$, for all $\gamma \in \operatorname{Dom}(p) \cup\{\alpha(\bar{y})\}$ and $m<\omega$. We let without loss of generality $\alpha(\bar{x}) \stackrel{\text { def }}{=} \alpha(\bar{y})$. 
Claim 6.4. Suppose that $p \in P_{\hat{\alpha}}^{\prime}$ and $\beta<\hat{\alpha}$, while $\operatorname{Dom}(p) \subseteq E V E N$. Suppose that $\bar{y}$ is a formal 0-canonical assignment (assignment, 0-canonical assignment) for $p$. Then $\bar{y}$ is a formal 0-canonical assignment (assignment, 0 -canonical assignment) for $p \nmid \beta$.

Proof of the Claim. Check, looking at (2), (5) and (6) of Definition 6.3. $\star 6.4$

Definition 6.5. By simultaneous induction on $\hat{\alpha} \leq \omega_{2}$ we define the following notions (a)-(d) and prove Lemma 6.6:

(a) For $a \in[\hat{\alpha}]^{\leq \aleph_{0}}$, sets $\mathrm{FE}_{n}(a)$ for $n<\omega$. The elements of $\mathrm{FE}_{n}(a)$ are called formal equivalence classes.

These are intended as formal $E_{n}$-equivalence classes.

(b) For $a \in[\hat{\alpha}]^{\leq \aleph_{0}}$, we define

(1) For $b \unlhd a \in[\hat{\alpha}]^{\leq \aleph_{0}}$, a function $F_{b, a}: \bigcup_{n<\omega} \mathrm{FE}_{n}(a) \rightarrow \bigcup_{n<\omega} \mathrm{FE}_{n}(b)$. $F$ is intended as a restriction to a smaller domain.

(2) Functions $\operatorname{Proj}_{n_{1}}^{n_{2}}(a): \mathrm{FE}_{n_{2}}(a) \rightarrow \mathrm{FE}_{n_{1}}(a)$, for $n_{1} \leq n_{2}<\omega$.

(c) For $a \in[\hat{\alpha}]^{\leq \aleph_{0}}$, we define functions $\operatorname{His}_{a}$ and Base ${ }_{a}$ by defining

(i) $\operatorname{His}_{a}(\hat{\Upsilon})$ for $\hat{\Upsilon} \in \bigcup_{n<\omega} \mathrm{FE}_{n}(a)$.

His stands for history.

(ii) $\operatorname{Base}_{a}(\hat{\Upsilon})$ for $\hat{\Upsilon} \in \bigcup_{n<\omega} \mathrm{FE}_{n}(a)$.

(d) For $p \in P_{\hat{\alpha}}^{\prime}$ with $\operatorname{Dom}(p) \subseteq E V E N$ and an assignment $\bar{x}$ for $p$ we define when $\bar{x}$ is a canonical assignment for $p$.

(e)

(I) For $p \in P_{\hat{\alpha}}$ and $n<\omega$ we define type $\frac{p, n}{\bar{x}}: R_{p}^{+} \rightarrow \mathrm{FE}_{n}(a)$, for $a \in[\hat{\alpha}]^{\leq \aleph_{0}}$. Here $\bar{x}$ is a canonical assignment for $p$. 
(II) For $p, \bar{x}$ as in (I), we define an equivalence relation $E_{\bar{x}}^{p, n}$ on $R_{p}^{+}$.

Lemma 6.6. Suppose that $p \in P_{\hat{\alpha}}^{\prime}$ with $\operatorname{Dom}(p) \subseteq E V E N$, and $\bar{x}$ is a canonical assignment for $p$. Then

$(1)^{\hat{\alpha}}$

$$
\mathfrak{B}_{p, \bar{x}} \stackrel{\text { def }}{=}\left(R_{p}^{+}, \bigcup_{m<\omega} A_{m}^{\alpha(\bar{x})}, E_{\bar{x}}^{p, n}\right)_{n<\omega}
$$

is a sweetness model.

$(2)^{\hat{\alpha}, \beta}$ Suppose that $\beta \leq \hat{\alpha}$. Then $\mathfrak{B}_{p \uparrow \beta, \bar{x}}<\mathfrak{B}_{p, \bar{x}}$.

$(3)^{\hat{\alpha}}$ For $b \unlhd a \in[\hat{\alpha}]^{\leq \aleph_{0}}$, we have that $F_{b, a}$ is a totally defined function.

We proceed to give the inductive definition and proof.

$\underline{\hat{\alpha}=0}$. In this case $p=\emptyset$ and $a=\emptyset$. We let

(a) $\mathrm{FE}_{n}(\emptyset) \stackrel{\text { def }}{=}\{\langle n, 0,0, \emptyset, \emptyset, \emptyset, \emptyset, \emptyset, \emptyset\rangle\}$ for $n<\omega$.

(b)

(1) $F_{\emptyset, \emptyset}$ is the identity.

(2) $\operatorname{Proj}_{n_{1}}^{n_{2}}(\emptyset): \mathrm{FE}_{n_{2}}(\emptyset) \rightarrow \mathrm{FE}_{n_{1}}(\emptyset)$ is given by

$$
\operatorname{Proj}_{n_{1}}^{n_{2}}(\emptyset)\left(\left\langle n_{2}, 0,0, \emptyset, \emptyset, \emptyset, \emptyset, \emptyset, \emptyset\right\rangle\right) \stackrel{\text { def }}{=}\left\langle n_{1}, 0,0, \emptyset, \emptyset, \emptyset, \emptyset, \emptyset, \emptyset\right\rangle,
$$

for $n_{1} \leq n_{2}<\omega$.

(c)

(i) $\operatorname{His}_{\emptyset}(\langle n, 0,0, \emptyset, \emptyset, \emptyset, \emptyset, \emptyset, \emptyset\rangle) \stackrel{\text { def }}{=}\left\{\left\langle n_{1}, 0,0, \emptyset, \emptyset, \emptyset, \emptyset, \emptyset, \emptyset, \emptyset\right\rangle: n_{1} \leq n\right\}$ for $n<\omega$.

(ii) $\operatorname{Base}_{\emptyset}(\langle n, 0,0, \emptyset, \emptyset, \emptyset, \emptyset, \emptyset, \emptyset\rangle) \stackrel{\text { def }}{=} \emptyset$.

(d) Any 0-canonical assignment for $\emptyset$ is a canonical assignment. 
(e)

(I) For $n<\omega$ we let type $\emptyset_{\emptyset}^{\emptyset, n}(\emptyset) \stackrel{\text { def }}{=}\langle n, 0,0, \emptyset, \emptyset, \emptyset, \emptyset, \emptyset, \emptyset\rangle$.

(II) For $n<\omega$, we let $\emptyset E_{\emptyset}^{\emptyset, n} \emptyset$.

Proof of the Lemma. [6.6, case $\hat{\alpha}=0]$. Trivial. $\star 6.6, \hat{\alpha}=0$

$\underline{\hat{\alpha}=\hat{\beta}+1}$. We first consider (a), (b) and (c) above. Fix $a \in[\hat{\alpha}] \leq \aleph_{0}$. Case $1 .[\hat{\beta}$ is odd] or $[\hat{\beta}$ is even $\& \hat{\beta} \notin a]$.

(a) For $n<\omega$, let $\mathrm{FE}_{n}(a) \stackrel{\text { def }}{=} \mathrm{FE}_{n}(a \cap \hat{\beta})$.

(1) For $b \unlhd a \in[\hat{\alpha}]^{\leq \omega_{2}}$ and $n<\omega$, we let $F_{b, a} \stackrel{\text { def }}{=} F_{b \cap \hat{\beta}, a \cap \hat{\beta}}$.

(2) For $n_{1} \leq n_{2}<\omega$, let $\operatorname{Proj}_{n_{1}}^{n_{2}}(a) \stackrel{\text { def }}{=} \operatorname{Proj}_{n_{1}}^{n_{2}}(a \cap \hat{\beta})$.

(c)

(i) $\operatorname{His}_{a} \stackrel{\text { def }}{=} \operatorname{His}_{a \cap \hat{\beta}}$.

(ii) Base $_{a} \stackrel{\text { def }}{=}$ Base $_{a \cap \hat{\beta}}$.

Case 2. (main case) $\hat{\beta}$ is even and $\hat{\beta} \in a$.

(a) For $n<\omega$,

$$
\mathrm{FE}_{n}(a) \stackrel{\text { def }}{=} \mathrm{FE}_{n}(a \cap \hat{\beta}) \cup\{\langle n, 1, \hat{\beta}, \Upsilon, t, w, u, \bar{k}, \bar{\varepsilon}\rangle:(*) \text { holds }\} \text {, }
$$

where for $(*)$ to hold it means that the following 6 items are satisfied:

1. $\Upsilon \in \mathrm{FE}_{k}(a \cap \hat{\beta})$ for some $k \geq n$ (the equivalence class of the initial segment),

2. $(\exists \hat{m})\left(t\right.$ is a subtree of $<\omega_{2}$ of height $\left.\hat{m}\right)$,

3. $w \subseteq\{0, \ldots, n-1\}$ (the places where there is an extension in the corresponding equivalence class) 
4. $u \subseteq\left\{(\eta, m): \eta \in{ }^{<n} 2\right.$ and $\left.m \in w\right\}$ (for $m \in w$, the witness that the $m$-th equivalence class in the enumeration produces to show that $\mathcal{T}_{\sim}^{r(\hat{\beta})}$ is nowhere dense),

5. $\bar{k}=\left\langle\left(k_{m}, \Upsilon_{m}\right): m \in w\right\rangle$ is such that

$$
(\forall m \in w)\left[k_{m}<\omega \& \Upsilon_{m} \in \mathrm{FE}_{k_{m}}(a \cap \hat{\beta})\right]
$$

(the sequence of $k$ 's for the equivalence classes of the projections),

6. $\bar{\varepsilon}$ is an increasing finite sequence with $\operatorname{Rang}(\bar{\varepsilon}) \subseteq a \cap E V E N$ (the coordinates where the equivalence class lives).

We let for $\hat{\Upsilon} \in \bigcup_{n<\omega} \mathrm{FE}_{n}(a)$,

$$
\hat{\Upsilon} \stackrel{\text { def }}{=}\left\langle n^{[\hat{\Upsilon}]}, o^{[\hat{\Upsilon}]}, \beta^{[\hat{\Upsilon}]}, \Upsilon^{[\hat{\Upsilon}]}, t^{[\hat{\Upsilon}]}, w^{[\hat{\Upsilon}]}, u^{[\hat{\Upsilon}]}, \bar{k}^{[\hat{\Upsilon}]}, \bar{\varepsilon}^{[\hat{\Upsilon}]}\right\rangle .
$$

(b)

(1) We define $F_{b, a}$ by cases:

Subcase 1. $a=b$.

$F_{b, a}$ is the identity.

Subcase 2. $b \neq a$ and $\hat{\Upsilon} \in \bigcup_{n<\omega} \mathrm{FE}_{n}(a \cap \hat{\beta})$.

$F_{b, a}(\hat{\Upsilon})=F_{b, a \backslash\{\hat{\beta}\}}(\hat{\Upsilon})$.

Subcase 3 . None of the Subcases 1 and 2 hold.

$F_{b, a}(\hat{\Upsilon})=F_{b, a \backslash\{\hat{\beta}\}}\left(\Upsilon^{[\hat{\Upsilon}]}\right)$.

(2) For $n_{1} \leq n_{2}<\omega$ we let $\left(\operatorname{Proj}_{n_{1}}^{n_{2}}(a)\right)\left(\Upsilon_{2}\right)=\Upsilon_{1}$ iff

$\underline{\text { Subcase 1. }} \Upsilon_{2} \in \mathrm{FE}_{n_{2}}(a \cap \hat{\beta})$ and $\Upsilon_{1}=\left(\operatorname{Proj}_{n_{1}}^{n_{2}}(a \cap \hat{\beta})\right)\left(\Upsilon_{2}\right)$.

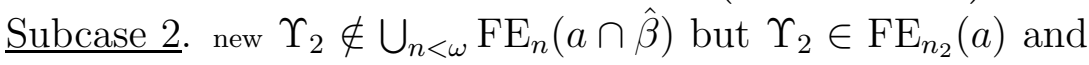
$\Upsilon_{1}$ satisfies $(\alpha)-(\eta)$ below, if possible:

$(\alpha) n^{\left[\Upsilon_{1}\right]}=n_{1}$, while $o^{\left[\Upsilon_{1}\right]}=1$ and $\beta^{\left[\Upsilon_{1}\right]}=\hat{\beta}$

$(\beta) \Upsilon^{\left[\Upsilon_{1}\right]}=\left(\operatorname{Proj}_{n_{1}}^{n_{2}}(a \cap \hat{\beta})\right)\left(F_{a \cap \hat{\beta}, a}\left(\Upsilon_{2}\right)\right)$,

$(\gamma) t^{\left[\Upsilon_{1}\right]}=t^{\left[\Upsilon_{2}\right]}$,

$(\delta) w^{\left[\Upsilon_{1}\right]}=w^{\left[\Upsilon_{2}\right]} \cap n_{1}$,

$(\varepsilon) u^{\left[\Upsilon_{1}\right]}=u^{\left[\Upsilon_{2}\right]} \cap\left\{(\eta, m): \eta \in \in^{<n_{1}} 2 \& m \in w^{\left[\Upsilon_{1}\right]}\right\}$,

$(\zeta) \bar{k}^{\left[\Upsilon_{1}\right]}=\bar{k}^{\left[\Upsilon_{2}\right]} \uparrow w^{\left[\Upsilon_{1}\right]}$, 


$$
\text { ( } \eta \bar{\varepsilon}^{\left[\Upsilon_{1}\right]}=\bar{\varepsilon}^{\left[\Upsilon_{2}\right]} .
$$

$\underline{\text { Subcase 3 }}$. If $F_{b, a}(\Upsilon)$ has not been defined by any of the two subcases above, we leave it undefined.

(c)

(i) $\operatorname{His}_{a}(\hat{\Upsilon})$ is given by

$$
\begin{aligned}
\operatorname{His}_{a}(\hat{\Upsilon})=\{\hat{\Upsilon}\} \cup & \bigcup_{n_{1} \leq n} \operatorname{His}_{a \backslash\{\hat{\Upsilon}\}}\left(F_{a \backslash\{\hat{\beta}\}, a}\left(\operatorname{Proj}_{n_{1}}^{\left.n^{[\hat{\Upsilon}}\right]}(a)\right)(\hat{\Upsilon})\right) \\
& \cup \bigcup_{m \in w^{[\hat{\Upsilon}]}} \operatorname{His}_{a \backslash\{\hat{\beta}\}}\left(\left(\operatorname{Proj}_{m}^{n^{[\hat{\Upsilon}]}}(a \backslash\{\hat{\beta}\})\right)\left(\Upsilon^{[\hat{\Upsilon}]}\right) .\right.
\end{aligned}
$$

(ii) $\operatorname{Base}_{a}(\hat{\Upsilon})=\left\{\left(\beta^{[\Upsilon]}, n_{1}\right): \Upsilon \in \operatorname{His}_{a}(\hat{\Upsilon}) \& n_{1} \leq n^{[\hat{\Upsilon}]}\right\}$.

We go on to define (d), (e) for the case $\hat{\alpha}=\hat{\beta}+1$.

(d) Let $\bar{x}$ be a 0 -canonical assignment for $p \in P_{\hat{\alpha}}^{\prime}$ (so we are assuming $\operatorname{Dom}(p) \subseteq E V E N)$.

Subcase $1 . \hat{\beta} \notin \operatorname{Dom}(p)$.

$\bar{x}$ is a canonical assignment for $p$ iff $\bar{x}$ is a canonical assignment for $p \uparrow \hat{\beta}$.

$\underline{\text { Subcase } 1 .} \hat{\beta} \in \operatorname{Dom}(p)$.

$\bar{x}$ is a canonical assignment for $p$ if $\bar{x}$ is a canonical assignment for $p \nmid \hat{\beta}$ and $\left\langle\bar{x} A_{m}^{\hat{\beta}}: m<\omega\right\rangle$ is an enumeration of all $E_{\bar{x} \uparrow \hat{\beta}}^{p \nmid \hat{\beta}, n}-$ equivalence classes for $n<\omega$.

(e) For $n<\omega$ and $\bar{x}$ a canonical assignment for $p$, we define the function type $\frac{p, n}{\bar{x}}: R_{p}^{+} \rightarrow \mathrm{FE}_{n}(a)$ by describing type $\frac{p, n}{\bar{x}}(r)$ for $r \in R_{p}^{+}$.

Subcase 1. $\hat{\beta} \notin \operatorname{Dom}_{p}^{*}(r)$ or $\hat{\beta} \notin \operatorname{Dom}(p)$.

We let type $\mathrm{e}_{\bar{x}}^{p, n}(r) \stackrel{\text { def }}{=} \operatorname{type}_{\bar{x}}^{p \uparrow \hat{\beta}, n}(r \vdash \hat{\beta})$.

Subcase 2. $\hat{\beta} \in \operatorname{Dom}_{p}^{*}(r)$.

We shall have type $\mathrm{e}_{\bar{x}}^{p, n}(r)=\hat{\Upsilon}$ for some $\hat{\Upsilon} \in \mathrm{FE}_{n}(a)$. We define $\hat{\Upsilon}$ by defining its 9 coordinates

$$
\left\langle n^{[\hat{\Upsilon}]}, o^{[\hat{\Upsilon}]}, \beta^{[\hat{\Upsilon}]}, \Upsilon^{[\hat{\Upsilon}]}, t^{[\hat{\Upsilon}]}, w^{[\hat{\Upsilon}]}, u^{[\hat{\Upsilon}]}, \bar{k}^{[\hat{\Upsilon}]}, \bar{\varepsilon}^{[\hat{\Upsilon}]}\right\rangle .
$$


We'll have $n^{[\hat{\Upsilon}]} \stackrel{\text { def }}{=} n, o^{[\hat{\Upsilon}]} \stackrel{\text { def }}{=} 1$, and $\beta^{[\hat{\Upsilon}]} \stackrel{\text { def }}{=} \hat{\beta}$. Furthermore, $t^{[\hat{\Upsilon}]}=t^{r(\hat{\beta})}$. Arriving to the heart of the matter,

$$
w^{[\hat{\Upsilon}]} \stackrel{\text { def }}{=}\left\{m<n: r\left\lceil\hat{\beta} \text { has an extension in }{ }^{\bar{x}} A_{m}^{\hat{\beta}}\right\},\right.
$$

while

$$
\begin{aligned}
& u^{[\hat{\Upsilon}]} \stackrel{\text { def }}{=}\left\{(\eta, m): \quad \eta \in^{<n} 2 \& m \in w^{[\hat{\Upsilon}]} \&\right. \\
& \text { for some } q \in \in^{\bar{x}} A_{m}^{\hat{\beta}} \text { we have } q \Vdash " \eta \notin \mathcal{T}_{\sim}^{r(\hat{\beta}) "} \text {, } \\
& \bar{k}^{[\hat{\Upsilon}]}=\left\langle\left\langle k_{m}(r \uparrow \hat{\beta}), \operatorname{type}_{\bar{x}}^{p \mid \hat{\beta}, m}(r \mid \hat{\beta})\right\rangle: m \in w^{[\hat{\Upsilon}]}\right\rangle \text {, where } \\
& k_{m}(r \uparrow \hat{\beta}) \stackrel{\text { def }}{=} \min \left\{k:\left(\forall q \in(r \uparrow \hat{\beta}) / E_{\bar{x}}^{p \uparrow \hat{\beta}, k}\right)\left(\exists q^{\prime} \in{ }^{\bar{x}} A_{m}^{\hat{\beta}}\right)\left(q^{\prime} \geq q\right)\right\} .
\end{aligned}
$$

The fact that such numbers $k_{m}(r \uparrow \hat{\beta})$ are well defined, is a part of the induction hypothesis (see Definition 2.13). Let

$$
k^{[\hat{\Upsilon}]} \stackrel{\text { def }}{=} \operatorname{Max}\left(\left\{k_{m}(r \mid \hat{\beta}): m<n\right\} \cup\{n\}\right) .
$$

We'll have

$$
\Upsilon^{[\hat{\Upsilon}]}=\operatorname{type}_{\bar{x}}^{p \nmid \hat{\beta}, k[\hat{\Upsilon}]}(r \vdash \hat{\beta}) .
$$

Finally, $\bar{\varepsilon}^{[\hat{\Upsilon}]}$ is the increasing list of $\operatorname{Dom}_{p}^{*}(r)$.

To see that the definition is well posed, notice that $r\left\lceil\hat{\beta} \in R_{p \nmid \hat{\beta}}^{+}\right.$.

(II) For $r^{\prime}, r^{\prime \prime} \in R_{p}^{+}$, we let

$$
r^{\prime} E_{\bar{x}}^{p, n} r^{\prime \prime} \text { iff } \operatorname{type}_{\bar{x}}^{p, n}\left(r^{\prime}\right)=\operatorname{type}_{\bar{x}}^{p, n}\left(r^{\prime \prime}\right) .
$$

Proof of the Lemma. [6.6, case $\hat{\alpha}=\hat{\beta}+1$ ].

Without loss of generality, $\beta=\hat{\beta}$. We prove $(2)^{\hat{\alpha}, \beta}$, and $(1)^{\hat{\alpha}}$ follows. By comparing with Definition 2.14 (which is [Sh 176, 7.6]), we can see that $\mathfrak{B}_{p, \bar{x}}$ is isomorphic to the canonical sweetness model on $R_{p \nmid \beta}^{+} * U M$ with respect to $\mathfrak{B}_{p \nmid \beta, \bar{x}}$. Notice that $U M$ is a homogeneous forcing notion. The conclusion follows from the Composition Lemma 2.17 (which is [Sh 176, 7.6-7.9]).

$(3)^{\hat{\alpha}}$ Follows from $(2)^{\hat{\alpha}, \beta} \cdot \star_{6.6}, \hat{\alpha}=\hat{\beta}+1$

$\underline{\hat{\alpha} \text { is a limit ordinal. }}$. 
(a) For $a \in[\hat{\alpha}]^{\leq \aleph_{0}}$, we consider two cases:

Case 1. $\sup (a)=\alpha<\hat{\alpha}$.

$\mathrm{FE}_{n}(a)$ is already defined by the induction hypothesis.

$\underline{\text { Case } 2 .} \sup (a)=\hat{\alpha}$.

We let $\mathrm{FE}_{n}(a) \stackrel{\text { def }}{=} \bigcup_{\alpha \in a} \mathrm{FE}_{n}(a \cap \alpha)$.

(b) We again consider two cases.

Case $1 . \sup (a)=\alpha<\hat{\alpha}$.

(1) For $b \unlhd a$, we have already defined $F_{b, a}$.

(2) Functions $\operatorname{Proj}_{n_{1}}^{n_{2}}(a)$ are defined by the induction hypothesis, for $n_{1} \leq n_{2}<\omega$.

$\underline{\text { Case } 2} \cdot \sup (a)=\hat{\alpha}$.

Subcase 1. $b=a$.

We define $F_{a, a}$ as the identity.

Subcase $2 . b \neq a$.

Suppose that $n<\omega$ and $\hat{\Upsilon} \in \mathrm{FE}_{n}(a)$. Let $\alpha<\hat{\alpha}$ be large enough such that $\hat{\Upsilon} \in \mathrm{FE}_{n}(a \cap \alpha)$ and $b \unlhd(a \cap \alpha)$. We let $F_{b, a}(\hat{\Upsilon}) \stackrel{\text { def }}{=} F_{b, a \cap \alpha}(\hat{\Upsilon})$.

(2) For $n_{1} \leq n_{2}<\omega$ and $\hat{\Upsilon} \in \mathrm{FE}_{n_{2}}(a)$, we define

$$
\left(\operatorname{Proj}_{n_{1}}^{n_{2}}(a)\right)(\hat{\Upsilon}) \stackrel{\text { def }}{=}\left(\operatorname{Proj}_{n_{1}}^{n_{2}}(a \cap \alpha)\right)(\hat{\Upsilon})
$$

if $\hat{\Upsilon} \in \mathrm{FE}_{n_{2}}(a \cap \alpha)$.

(c)

(i) For $\hat{\Upsilon} \in \bigcup_{n<\omega} \operatorname{FE}_{n}(a)$, we define $\operatorname{His}_{a}(\hat{\Upsilon}) \stackrel{\text { def }}{=} \operatorname{His}_{a \cap \alpha}(\hat{\Upsilon})$ for any $\alpha<\hat{\alpha}$ such that $\hat{\Upsilon} \in \bigcup_{n<\omega} \operatorname{FE}_{n}(a \cap \alpha)$.

(ii) For $\hat{\Upsilon} \in \bigcup_{n<\omega} \operatorname{FE}_{n}(a)$, we let $\operatorname{Base}_{a}(\hat{\Upsilon}) \stackrel{\text { def }}{=} \operatorname{Base}_{a \cap \alpha}(\hat{\Upsilon})$ for $\alpha$ such that $\hat{\Upsilon} \in \bigcup_{n<\omega} \operatorname{FE}_{n}(a \cap \alpha)$.

(d) $\bar{x}$ is a canonical assignment for $p \in P_{\hat{\alpha}}^{\prime}$ iff for all $\beta<\hat{\alpha}$ we have that $\bar{x}$ is a canonical assignment for $p \uparrow \beta$. 
(e)

(I) Suppose that $n<\omega$. For $r \in R_{p}^{+}$we let

$$
\operatorname{type}_{\bar{x}}^{p, n}(r) \stackrel{\text { def }}{=} \operatorname{type}_{\bar{x}}^{p \nmid \alpha, n}(r \nmid \alpha)
$$

for any $\alpha<\hat{\alpha}$ such that $\operatorname{Dom}_{p}^{*}(r) \subseteq \alpha$.

(II) For $n<\omega$ and $r^{\prime}, r^{\prime \prime} \in R_{p}^{+}$, we let

$$
r^{\prime} E_{\bar{x}}^{p, n} r^{\prime \prime} \operatorname{iff}\left(r^{\prime}\lceil\alpha) E_{\bar{x}}^{p \nmid \alpha, n}\left(r^{\prime \prime} \uparrow \alpha\right)\right.
$$

for any $\alpha<\hat{\alpha}$ such that $\operatorname{Dom}_{p}^{*}\left(r^{\prime}\right) \cup \operatorname{Dom}_{p}^{*}\left(r^{\prime \prime}\right) \subseteq \alpha$.

As a part of the inductive definition in the case $\hat{\alpha}$ a limit ordinal, we prove the following

Observation 6.7. Objects in items (a)-(e) above are well defined.

Proof of the Observation. We have to check several spots where the definition in the case of $\hat{\alpha}$ limit might run into a contradiction. We start by (b) Case 2(1), Subcase 2. We assume that $\alpha_{1} \leq \alpha_{2}<\hat{\alpha}$, while $\hat{\Upsilon} \in \mathrm{FE}_{n}\left(a \cap \alpha_{1}\right) \cap \mathrm{FE}_{n}\left(a \cap \alpha_{2}\right)$, and $b \unlhd a \cap \alpha_{1}$. We can prove by induction on $\alpha \in\left[\alpha_{1}, \alpha_{2}\right]$ that $F_{b, a \cap \alpha_{1}}(\hat{\Upsilon})=F_{b, a \cap \alpha}(\hat{\Upsilon})$. Note the definition in the case that $\hat{\alpha}$ is a successor ordinal, item (b)(1), Subcase 2 of Case 2.

We move on to (b), Case 2(2).

Suppose that $\alpha_{1} \leq \alpha_{2}<\hat{\alpha}$ and $\hat{\Upsilon} \in \mathrm{FE}_{n_{2}}\left(a \cap \alpha_{1}\right) \cap \mathrm{FE}_{n_{2}}\left(a \cap \alpha_{2}\right)$. We can prove by induction on $\alpha \in\left[\alpha_{1}, \alpha_{2}\right]$ that

$$
\left(\operatorname{Proj}_{n_{1}}^{n_{2}}\left(a \cap \alpha_{1}\right)\right)(\hat{\Upsilon})=\left(\operatorname{Proj}_{n_{1}}^{n_{2}}(a \cap \alpha)\right)(\hat{\Upsilon})
$$

Observe the way the definition is set up in Subcase 1. of Case 2. (b)(2) of the definition for the case of $\hat{\alpha}$ being a successor ordinal.

We go to item (c), part (i), which is proved similarly, observing the set up of the definition in the case of $\hat{\alpha}$ being a successor ordinal, Case 2, item (c) (i). Similarly for item (c), part (ii). 
We still have to check items (d) and (e), which is done in a similar fashion. $\star 6.7$

Proof of the Lemma. [6.6, case $\hat{\alpha}$ a limit].

First suppose $\operatorname{cf}(\hat{\alpha})=\aleph_{0}$. We prove $(2)^{\hat{\alpha}, \beta}$ for a given $\beta \leq \hat{\alpha}$. Without loss of generality, $\beta<\hat{\alpha}$. Let $\left\langle\alpha_{n}: n<\omega\right\rangle$ be an increasing sequence of ordinals with $\alpha_{0}=\beta$ and $\sup _{n<\omega} \alpha_{n}=\hat{\alpha}$. Considering $\mathfrak{B}_{p \nmid \alpha_{n}, \bar{x}}(n<\omega)$, we finish by the induction hypothesis and Fact 2.18 .

If $\underline{\mathrm{cf}(\hat{\alpha}) \geq \aleph_{1}}$, the conclusion follows by the induction hypothesis.

This ends the inductive definition.

Claim 6.8. Suppose that $\alpha \leq \omega_{2}$ and $p \in P_{\alpha}^{\prime}$. Then

$(1)^{\alpha}$ The function $i$ given by $i(r)=r\lceil E V E N$ is a complete embedding of $R_{p}$ into $R_{p \mid E V E N}$.

$(2)^{\alpha} R_{p\lceil E V E N}$ is a complete suborder of $R_{p}$.

Proof of the Claim. We prove $(1)^{\alpha}-(2)^{\alpha}$ together, by induction on $\alpha$. The proof is straightforward. $\star 6.8$

Notation 6.9. For $\alpha \leq \omega_{2}$, we let $P_{\alpha}^{\prime}\left\lceil E V E N \stackrel{\text { def }}{=}\left\{p \nmid E V E N: p \in P_{\alpha}^{\prime}\right\}\right.$.

Definition 6.10. Suppose that $\alpha \leq \omega_{2}$ and $p \leq_{\text {pr }} q \in P_{\alpha}^{\prime}$. Further suppose that $p^{\prime}=p\left\lceil E V E N\right.$ and $q^{\prime}=q\left\lceil E V E N\right.$, while $\operatorname{Dom}\left(q^{\prime}\right)=\operatorname{Dom}\left(p^{\prime}\right)$.

Notice: $q^{\prime}$ and $p^{\prime}$ are not necessarily the same name, as the names of $q(\beta)$ for $\beta$ even might depend on coordinates of $q$ outside of $\operatorname{Dom}(p)$.

By induction on $\alpha$ we define $(\mathrm{A})^{\alpha}$ and prove $(\mathrm{B})^{\alpha}$ below: 
$(\mathrm{A})^{\alpha}$ Suppose that $\bar{x}$ is a canonical assignment for $q^{\prime}$ with $\alpha(\bar{x}) \leq \alpha$. We define $\bar{x}: p^{\prime}$ by letting

$$
\bar{x}: p^{\prime} \stackrel{\text { def }}{=}\left\langle\bar{x}: p^{\prime} A_{m}^{\beta}: \beta \in\left[\operatorname{Dom}(\bar{x}) \cap\left(\operatorname{Dom}\left(p^{\prime}\right)\right] \cup\{\alpha(\bar{x})\}\right)\right\rangle,
$$

and for $\beta \in \operatorname{Dom}\left(\bar{x}: p^{\prime}\right)$, for the unique $n<\omega$ and $\Upsilon \in \mathrm{FE}_{n}\left(\operatorname{Dom}\left(p^{\prime}\right)\right)$ such that

$$
{ }^{\bar{x}} A_{m}^{\beta}=\left\{z \in R_{q^{\prime} \uparrow \beta}^{+}: \operatorname{type}_{\bar{x}}^{q^{\prime} ґ \beta, n}(z)=\Upsilon\right\},
$$

we have

$$
\bar{x}: p^{\prime} A_{m}^{\beta} \stackrel{\text { def }}{=}\left\{\check{z} \in R_{p^{\prime} \uparrow \beta}^{+}: \operatorname{type}_{\bar{x}: p^{\prime}}^{p^{\prime} \uparrow \beta}(\check{z})=\Upsilon\right\} .
$$

$(\mathrm{B})^{\alpha}$

Claim 6.11. Suppose that $\bar{x}$ is a canonical assignment for $q^{\prime}$ with $\alpha(\bar{x}) \leq \alpha$. Then $\bar{x}: p^{\prime}$ is a canonical assignment for $p^{\prime}$.

Proof of the Claim. Check Definition 6.5. $\star 6.11$

\section{$7 \quad$ More partial orders}

Claim 7.1. Suppose that $\alpha \leq \omega_{2}$, while $p \leq p^{*} \in P_{\alpha}^{\prime}$ and $q_{1}, q_{2} \in R_{p}$ are such that $q_{1}, q_{2} \leq p^{*}$. Then there is $p^{* *} \geq p^{*}$ and $q^{*} \in R_{p}$ such that $q_{1}, q_{2} \leq$ apr $q^{*}$ and $q^{*} \leq p^{* *}$, and $p^{* *} \in P_{\alpha^{\prime}}$.

Proof of the Claim. The proof is by induction on $\alpha$. The eventful case of the induction is when $\alpha=\beta+1$ for some even $\beta \in \operatorname{Dom}(p)$ such that

$$
\neg\left(q_{1} \uparrow \beta \Vdash " q_{1}(\beta)=p(\beta) " \text { and } q_{2}\left\lceil\beta \Vdash " q_{2}(\beta)=p(\beta) "\right) .\right.
$$

We can find $p^{\prime} \geq p^{*} \uparrow \beta$ in $P_{\beta}^{\prime}$ which forces a value to all

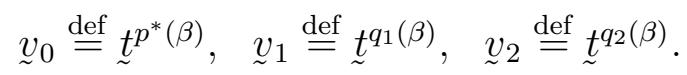

By the induction hypothesis, possibly extending $p^{\prime}$, there is $q^{\prime} \in R_{p \nmid \beta}$ such that $q_{1} \uparrow \beta, q_{2} \uparrow \beta \leq$ apr $q^{\prime}$ and $q^{\prime} \leq p^{\prime}$. We know that $q^{\prime} \uparrow \beta$ forces the 
existence of a predense set $\underset{\sim}{J}$ in $R_{p \nmid \beta}$ such that each condition in $\underset{\sim}{J}$ forces all of the above values. Possibly increasing $p^{\prime}$ we can assume that there are $r_{0}, r_{1}, r_{2} \in R_{p \nmid \beta}$ forcing a value to $v_{0}, v_{\sim}, v_{2}$ respectively, and all below $p^{\prime}$. By the induction hypothesis, possibly extending $p^{\prime}$ again, there is $q \in R_{p \nmid \beta}$ which is above $q^{\prime}$ and $r_{0}-r_{2}$, and below $p^{\prime}$. Let

$$
q^{*} \stackrel{\text { def }}{=} \underset{\left.\left.\mathcal{T}^{q_{1}(\beta)} \cup\left\{\mathcal{T}^{q_{2}(\beta)}\right)\right)\right\},}{\cup\left(\beta,\left(\left\{\eta: q \Vdash " \eta t^{q_{1}(\beta)} \cup t_{\sim}^{q_{2}(\beta)} "\right\},\right.\right.}
$$

and $p^{* *} \stackrel{\text { def }}{=} p^{\prime} \cup\left\{\left(\beta, q^{*}(\beta)\right)\right\}$. We need to check that $q^{*}(\beta)$ is simple above $q$ (so above $p^{\prime}$ ), which follows as $q_{l}(\beta)$ is simple above $q_{l} \uparrow \beta$ for $l=1,2$. $\star$. .

Corollary 7.2. If $\alpha, p, q_{1}, q_{2}$ are as in Claim 7.1, then $q_{1}, q_{2}$ are compatible in $P_{\alpha}^{\prime}$ iff $q_{1}, q_{2}$ are compatible in $R_{p}$.

Definition 7.3. Suppose that $\alpha \leq \omega_{2}$ and $u \subseteq \operatorname{Dom}(p)$. We define

(1) $G R_{p, u} \stackrel{\text { def }}{=}\left\{q \in R_{p}: \operatorname{Dom}_{p}^{*}(q) \cap u\right\}=\emptyset$.

(2) $R_{p, u}^{+} \stackrel{\text { def }}{=}\left\{q \in R_{p}^{+}: \operatorname{Dom}_{p}^{*}(q) \subseteq u\right\}$.

We make $G R_{p, u}$ and $R_{p, u}^{+}$into partial orders by letting them inherit the order from $R_{p}$.

Claim 7.4. Suppose that $\alpha \leq \omega_{2}$, while $p \in P_{\alpha}^{\prime}, u \subseteq \operatorname{Dom}(p)$ and $r \in R_{p, u}^{+}$ and $s \in G R_{p, u}$. Then the following is a well defined condition in $R_{p}$ : for $\beta \in \operatorname{Dom}(p)$ we let

$$
(r \cup s)(\beta) \stackrel{\text { def }}{=} \begin{cases}r(\beta) & \text { if } \beta \in \operatorname{Dom}_{p}^{*}(r) \\ s(\beta) & \text { if } \beta \in \operatorname{Dom}_{p}^{*}(s) \\ p(\beta) & \text { otherwise. }\end{cases}
$$

In addition, $r \cup s \geq_{\text {apr }} r, s$. 
Proof of the Claim. The proof is by induction on $\alpha$, and the only interesting case is when $\alpha=\beta+1$ for some even $\beta \in \operatorname{Dom}(p)$. Note that exactly one of the clauses in the definition of $(r \cup s)(\beta)$ applies. Let us work with the first one, as the other cases are similar.

Hence $\beta \in \operatorname{Dom}_{p}^{*}(r)$ and $(r \cup s)\lceil\beta \Vdash "(r \cup s)(\beta)=r(\beta)$ ". So we have that $(r \cup s)\left|\beta \geq_{\text {apr }} r\right| \beta$ and $r \geq_{\text {pr }} r \mid \beta$. By Claim $4.16(2)^{\alpha}$, $r \cup s=[(r \cup s) \vdash \beta+r]$ is well defined, and the rest of the Claim is easily verified. $\star$. 4

Notation 7.5. We extend our definition of " $r+s$ " from 4.16 to apply also to $r, s$ as in Claim 7.4, letting $r+s \stackrel{\text { def }}{=} r \cup s$.

Definition 7.6. Suppose that $Q$ is a forcing notion and $M \prec\left(H(\chi), \in,<^{*}\right)$ is countable. We say that an increasing sequence $\bar{s}=\left\langle s_{n}: n<\omega\right\rangle$ of conditions in $Q \cap M$ is a generic enough sequence for $(Q, M)$ iff for every formula $\varphi$ with parameters in $M$, there are infinitely many $n$ such that

$(\alpha)$ Either there is no $s \geq s_{n}$ in $Q$ such that $\varphi(s)$ holds, or

( $\beta) \varphi\left(s_{n+1}\right)$.

Claim 7.7. Suppose $\alpha \leq \omega_{2}$, while $p \in P_{\alpha}^{\prime}$ and $u \subseteq \operatorname{Dom}(p)$.

(1) Suppose that $s \in G R_{p, u}$ and $r \in R_{p}^{+}$are compatible. Then there are $s^{\prime} \in G R_{p, u}$ and $r^{\prime} \in R_{p, u}^{+}$such that $s \leq s^{\prime}$ and $r \leq r^{\prime}+s^{\prime}$.

(Hence $r$ is compatible with every $s^{\prime \prime} \geq s^{\prime}$ for which $s^{\prime \prime} \in G R_{p, u}$.)

(2) Suppose that $\left\{u, p, \bar{Q}, \bar{Q}^{\prime}, \alpha\right\} \subseteq M \prec\left(H(\chi), \in,<^{*}\right)$ is countable and $\bar{s}=\left\langle s_{n}: n<\omega\right\rangle$ is a generic enough sequence for $\left(G R_{p, u}, M\right)$. Further suppose $\gamma \in M \cap(\alpha+1)$ and $r \in R_{p \mid \gamma}^{+} \cap M$ is compatible with all $s_{n}$. Then there is $r^{\prime} \in R_{p \nmid \gamma, u}^{+} \cap M$ such that for all large enough $n$ we have $r \leq r^{\prime}+s_{n}$. 
Proof of the Claim. (1) The proof is by induction on $\alpha$. The interesting case is when $\alpha=\beta+1$ for some even $\beta \in \operatorname{Dom}(p)$.

By the induction hypothesis, there are $q^{\prime} \in R_{p \nmid \beta, u \cap \beta}^{+}$and $t^{\prime} \in G R_{p \nmid \beta, u \cap \beta}$ such that $s \uparrow \beta \leq t^{\prime}$ and $r \uparrow \beta \leq q^{\prime}+t^{\prime}$.

We first work in the case that $\left(q^{\prime}+t^{\prime}\right) \Vdash " r(\beta) \geq s(\beta)$ ". If $\beta \notin u$ this means that $s\lceil\beta \Vdash " s(\beta)=p(\beta)$ " and $r \uparrow \beta \Vdash " r(\beta)=p(\beta)$ ". We define $r^{\prime} \stackrel{\text { def }}{=} q^{\prime}+p$, which is well defined by Claim $4.16(2)^{\alpha}$. It is easily seen that $r^{\prime} \in R_{p, u}^{+}$. Similarly we define $s^{\prime} \stackrel{\text { def }}{=} t^{\prime}+p$, and check that $r^{\prime}, s^{\prime}$ are as required.

If $\beta \in u$, we define $r^{\prime} \stackrel{\text { def }}{=} q^{\prime}+r$ (note that $q^{\prime} \in R_{r \uparrow \beta}^{+}$), and $s^{\prime} \stackrel{\text { def }}{=} t^{\prime}+p$, and check that $r^{\prime}, s^{\prime}$ are as required.

It remains to be seen what happens in the case that it is not true that $\left(q^{\prime}+t^{\prime}\right) \Vdash " r(\beta) \geq s(\beta)$ ". As $r$ and $s$ are compatible, we can by Claim 7.1 find $z \in R_{p}$ such that $z \geq r, s$. By Claim 6.2, we can find $z^{+} \geq^{+} z$, hence $z^{+} \in R_{p}^{+}$and $z^{+} \geq s$. Now we can apply the first part of the proof to $z$ and $s$, and derive the desired conclusion.

If $s^{\prime \prime} \geq s^{\prime}$ and $s^{\prime \prime} \in G R_{p, u}$, then $r \leq r+s^{\prime \prime}$ and $s^{\prime \prime} \leq r+s^{\prime \prime}$, so $r, s^{\prime \prime}$ are compatible.

(2) Without loss of generality, $\alpha=\gamma$. Let

$$
I \stackrel{\text { def }}{=}\left\{s^{\prime} \in G R_{p, u}:\left(\exists r^{\prime} \in R_{p, u}^{+}\right)\left(r \leq r^{\prime}+s^{\prime}\right)\right\} .
$$

Hence $I \in M$. Let $n$ be such that when choosing $s_{n}$ we have asked if there was $s^{\prime} \geq s_{n}$ with $s^{\prime} \in I$, and if possible we chose $s_{n+1}$ to be some such $s^{\prime}$. (In other words, either there is no $s^{\prime} \geq s_{n}$ with $s^{\prime} \in I$, or $s_{n+1} \in I$.) As $r, s_{n}$ are compatible, by (1), we have chosen $s_{n+1}$ so that for some $r^{\prime} \in R_{p, u}^{+}$we have $r \leq r^{\prime}+s_{n}$ 太西

Definition 7.8. Suppose that $\alpha \leq \omega_{2}$ and $\left\langle s_{n}: n<\omega\right\rangle$ and $u, M$ are as above. Further suppose that $r \in R_{s_{0}\lceil\alpha}^{+}$is compatible with all $s_{n}$ and $r \in M$.

$$
\begin{aligned}
& \text { We define } \operatorname{Dom}(r / \bar{s}) \stackrel{\text { def }}{=} \operatorname{Dom}_{s_{0} \nmid \alpha}^{*}(r) \cap u \text {, and for } \beta \in \operatorname{Dom}(r / \bar{s}) \\
& \qquad(r / \bar{s})(\beta) \stackrel{\text { def }}{=}\left(t^{r(\beta)}, \bigcup_{n<\omega}\left\{\eta \in^{<\omega} 2:\left(r+s_{n}\right) \vdash \beta \Vdash " \eta \in_{\sim}^{\left.\mathcal{T}^{r(\beta)}\right) "}\right\}\right) .
\end{aligned}
$$


(2) Suppose that $n<\omega$, we define $\operatorname{Dom}\left(r / s_{n}\right) \stackrel{\text { def }}{=} \operatorname{Dom}_{s_{0}\lceil\alpha}^{*}(r) \cap u$ and for $\beta \in \operatorname{Dom}\left(r / s_{n}\right)$

$$
\left(r / s_{n}\right)(\beta) \stackrel{\text { def }}{=}\left(t^{r(\beta)}, \bigcup_{m<n}\left\{\eta \in \epsilon^{<\omega} 2:\left(r+s_{m}\right) \mid \beta \Vdash " \eta \in \mathcal{T}^{r(\beta)} "\right\}\right) .
$$

(3) Suppose that $I$ is a subset of $P_{\alpha}$. We let

$$
I / \bar{s} \stackrel{\text { def }}{=}\{q / \bar{s}: q \in I \& q / \bar{s} \text { defined }\} .
$$

Definition 7.9. For $\alpha \leq \omega_{2}, p \in P_{\alpha}^{\prime}\left\lceil E V E N\right.$ and $q_{1}, q_{2} \in R_{p}^{+}$which are compatible, we define $q_{1} \oplus q_{2}$ by letting

$$
\left(q_{1} \oplus q_{2}\right)(\beta) \stackrel{\text { def }}{=}\left(t^{q_{1}(\beta)} \cup t^{q_{2}(\beta)}, \mathcal{T}^{q_{1}(\beta)} \cup \mathcal{T}^{q_{2}(\beta)}\right) .
$$

Remark 7.10. If $p, q_{1}, q_{2}$ are as above, then $q_{1} \oplus q_{2}$ is the lub of $q_{1}, q_{2}$ in $R_{p}$. (this can be proved by induction on $\alpha$ ).

Claim 7.11. Suppose $\alpha \leq \omega_{2}$ and $\bar{s}=\left\langle s_{n}: n<\omega\right\rangle$, and $u, M$ are as above.

$(1)^{\alpha}$ If $r \in R_{s_{0}\lceil\alpha}^{+} \cap M$ is compatible with all $s_{n}$, then $r / \bar{s} \in P_{\alpha}^{\prime}$, and for all large enough $n$ we have $r / s_{n} \in P_{\alpha}^{\prime}$.

$(2)^{\alpha}$ Given $q, r \in R_{s_{0}\lceil\alpha}^{+} \cap M$ compatible with all $s_{n}$, then

$$
[q / \bar{s} \geq r / \bar{s}] \Longleftrightarrow\left[\left(\forall^{*} n\right)\left(q+s_{n} \geq r+s_{n}\right)\right] .
$$

$(3)^{\alpha}$ Suppose that $\underset{\sim}{I} \in M$ and $r \in R_{s_{0}\lceil\alpha}^{+} \cap M$ is compatible with all $s_{n}$, while

$$
r \Vdash \text { "I } I \text { countable predense } \subseteq R_{r} . "
$$

Then

$$
r / \bar{s} \Vdash \text { "I } I / \bar{s} \text { countable predense } \subseteq R_{r / \bar{s}} \text { " }
$$


$(4)^{\alpha}$ Suppose that $\alpha=\beta+1$ for some $\beta \in \operatorname{Dom}(r / \bar{s})$. Further suppose that $r \in R_{s_{0}\lceil\alpha}^{+} \cap M$ and $q \in R_{s_{0}\lceil\beta}^{+} \cap M$ are compatible with all $s_{n}$, while $n<\omega$ and

$$
r \uparrow \beta \Vdash \text { " } q \text { determines } r(\alpha) \text { to degree } n . "
$$

$\underline{\text { Then }}$

$$
(r / \bar{s}) \uparrow \beta \Vdash " q / \bar{s} \text { determines }(r / \bar{s}(\alpha)) \text { to degree } n . "
$$

For $r, q$ as above, if $t$ is such that $q \Vdash$ "Tํㄱ ${ }^{r(\beta)} \cap<n 2=t$ ", then

$$
q / \bar{s} \Vdash " \mathcal{T}^{(r / \bar{s})(\beta)} \cap{ }^{<n} 2=t " .
$$

$(5)^{\alpha}$ Suppose that $\bar{x}$ is a canonical assignment for $s_{0} \vdash E V E N$. Further

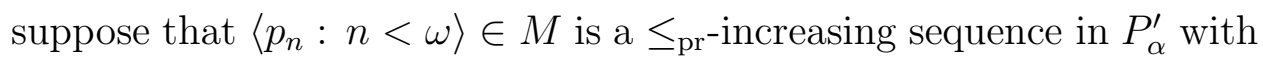
limit $p$, such that $p \leq_{\mathrm{pr}} s_{0}$ and $\operatorname{Dom}(p)=u$. Then for every $n<\omega$

$$
\left(\forall^{*} l<\omega\right) \begin{aligned}
& {\left[\operatorname{type}_{\bar{x}}^{s_{0} \mid(E V E N \cap \alpha), n}\left(\left(r+s_{l}\right) \uparrow(E V E N \cap \alpha)\right)=\right.} \\
& \operatorname{type}_{\bar{x}: p_{l}}^{p_{l}(E V E N \cap \alpha), n}\left(\left(r / \bar{s}+p_{l}\right)\lceil(E V E N \cap \alpha))\right] .
\end{aligned}
$$

Proof of the Claim. We prove the claim by induction on $\alpha$, proving $(1)^{\alpha}$ $(5)^{\alpha}$ simultaneously. The only eventful case of the induction is when $\alpha=\beta+1$ for some $\beta$ even.

$(1)^{\alpha}$ By $(1)^{\beta}$, we have that $(r / \bar{s})\left\lceil\beta \in P_{\beta}^{\prime}\right.$. Without loss of generality, $\beta \in \operatorname{Dom}_{s_{0}\lceil\alpha}^{*}(r) \cap u$. Given $G$ which is $P_{\beta}$-generic and contains $(r / \bar{s})\lceil\beta$. We have

(a) $\mathcal{T}_{G}^{(r / \bar{s})(\beta)} \cap<\mathrm{ht}\left(t^{r(\beta)}\right) 2=t^{r(\beta)}$, as the corresponding statement about $\mathcal{T}^{r(\beta)}$ is forced by each $\left(r+s_{n}\right)\lceil\beta$.

(b) Similarly, $\mathcal{T}_{G}^{(r / \bar{s})(\beta)}$ is perfect.

(c) We show that $\mathcal{T}_{G}^{(r / \bar{s})(\beta)}$ is nowhere dense.

Given $\eta \in{ }^{<\omega} 2$ and $n^{*}$ such that $\left(r+s_{n^{*}}\right) \mid \beta \Vdash " \eta \in \mathcal{T}_{\sim}^{r(\beta)}$ ". At some stage $n \geq n^{*}$ we have asked if there is $s \geq s_{n}$ with $s \in G R_{p, u} \cap M$ such that for some $q \geq r+s_{n}$ with $q \in R_{p}^{+}$, and

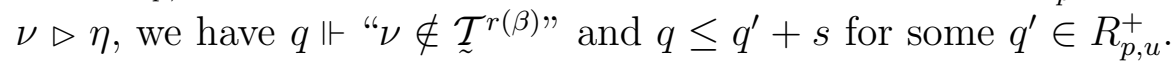


By Claim 7.7(1), there was some such $s$ which was chosen as $s_{n+1}$. In particular $q+s_{m} \geq r+s_{m}$ for any $m \geq n+1$. So for no $m$ can

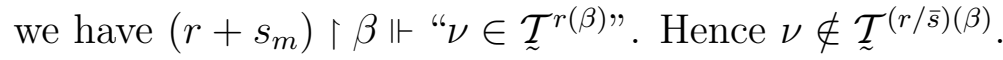

(d) We show that $r / \bar{s}$ is simple above $(r / \bar{s}) \uparrow \beta$.

Let $\bar{\sim}=\left\langle I_{n}: n<\omega\right\rangle$ exemplify that $r(\beta)$ is simple above $r \mid \beta$. Without loss of generality, $\underset{\sim}{I} \in M$. By $(3)^{\beta}+(4)^{\beta}$ we have that $\left\langle I_{n} / \bar{s}: n<\omega\right\rangle$ exemplify that $r / \bar{s}$ is simple above $(r / \bar{s}) \uparrow \beta$.

$(2)^{\alpha}$ Again without loss of generality we have $\beta \in \operatorname{Dom}(r / \bar{s})$. First we prove the direction from right to left.

By the induction hypothesis, $(q / \bar{s}) \uparrow \beta \geq(r / \bar{s}) \uparrow \beta$. By the assumption, $t^{q(\beta)} \supseteq t^{r(\beta)}$. Suppose that for some $n$ large enough and $\eta \in{ }^{<\omega} 2$, we have

$$
\left(r+s_{n}\right)\left\lceil\beta \Vdash " \eta \in \mathcal{T}_{\sim}^{r(\beta)} "\right.
$$

As $q+s_{n} \geq r+s_{n}$, we have

$$
\left(q+s_{n}\right)\left\lceil\beta \Vdash " \eta \in \mathcal{T}^{q(\beta) "} .\right.
$$

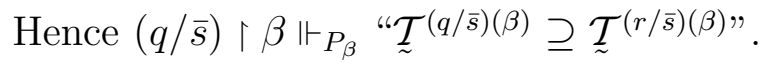

Suppose that for some $\eta \in<^{<\mathrm{ht}\left(t^{r(\beta)}\right)} 2$ and $n$ large enough we have $\left(q+s_{n}\right) \mid \beta \Vdash " \eta \in \mathcal{T}_{\sim}^{q(\beta)}$. As $q+s_{n} \geq r+s_{n}$, we have $\eta \in t^{r(\beta)}$.

In the direction from left to right, by the induction hypothesis we have that

$$
\left(\forall^{*} n\right)\left[\left(q+s_{n}\right) \vdash \beta \geq\left(r+s_{n}\right) \uparrow \beta\right] .
$$

By the assumption, $t^{q(\beta)} \supseteq t^{r(\beta)}$. Suppose that for some $n^{*}$ large enough, and $\eta \in{ }^{<\omega} 2$, we have $\left(q+s_{n^{*}}\right) \uparrow \beta \Vdash " \eta \in \mathcal{T}^{r(\beta)}$ ". Let $m \stackrel{\text { def }}{=} \lg (\eta)$. Let $\underset{\sim}{I}=\left\langle I_{n}: n<\omega\right\rangle \in M$ exemplify that $r(\tilde{\beta})$ is simple above $r \uparrow \beta$. Hence, it is forced by $r \uparrow \beta$ that for some $z \in I_{m} \cap M$ which

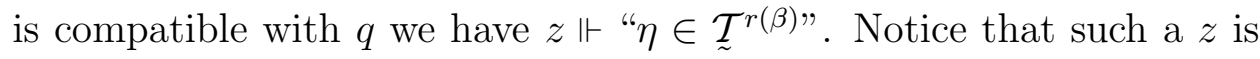
compatible with every $s_{n}$. Hence $z / \bar{s}$ is defined and by $(4)^{\beta}$ we have $z / \bar{s} \Vdash " \eta \eta \mathcal{T}^{(r / s)(\beta) "}$. We also have that $z / \bar{s} \geq(q\lceil\beta) / \bar{s} \geq(r \mid \beta) / \bar{s}$, and

$$
q\left\lceil\beta / \bar{s} \Vdash " \eta \in \mathcal{T}^{r / \bar{s}(\beta)} \Longrightarrow \eta \in \mathcal{T}^{q / \bar{s}(\beta) "} .\right.
$$


So $z / \bar{s} \Vdash " \eta \in \mathcal{T}^{q / \bar{s}(\beta) "}$. As $z / \bar{s}$ and $q+s_{n}$ are compatible for all large enough $n$, it must be that for some $n^{*}$ large enough

$$
\left(q+s_{n^{*}}\right)\left\lceil\beta \Vdash " \eta \in \mathcal{T}^{q(\beta)},\right.
$$

(by the genericity of $\bar{s}$ ).

Now suppose that for some $\eta \in^{<\mathrm{ht}\left(t^{r(\beta)}\right)} 2$ and $n$ large enough we have $\left(q+s_{n}\right)\left\lceil\beta \Vdash " \eta \in \mathcal{T}_{\sim}^{q(\beta)}\right.$ ". Hence $\eta \in \mathcal{T}_{\sim}^{q / \bar{s}(\beta)}$, so, as $q / \bar{s} \geq r / \bar{s}$, it must be that $\eta \in t^{r(\beta)}$.

$(3)^{\alpha}$ Certainly $r / \bar{s}$ forces $I / \bar{s}$ to be countable, and by $(2)^{\alpha}$ we also know that $r / \bar{s} \Vdash " I I / \bar{s} \subseteq R_{r / \bar{s}}$ ".

We show that

$$
r / \bar{s} \Vdash \text { "I } / / \bar{s} \text { predense } \subseteq R_{r / \bar{s}} " .
$$

Let $\underset{\sim}{I}=\left\{q_{l}: l<\omega\right\}$. Suppose that $z^{*} \in R_{r / \bar{s}}$, and we wish to show that $z^{*}$ is compatible with some $q_{l} / \bar{s}$. Without loss of generality, $z^{*} \in R_{r / \bar{s}}^{+}$. If $q_{l} / \bar{s}$ is defined and $z^{*} \uparrow \beta$ is compatible with $\left(q_{l} / \bar{s}\right) \uparrow \beta$, the only way that $z^{*}$ and $q_{l} / \bar{s}$ can turn out to be incompatible, is that one of the following happens:

(i) Neither is $t^{z(\beta)}$ an end extension of $t^{(q / \bar{s})(\beta)}$, nor the other way around,

(ii) or $t^{z(\beta)}$ is an end extension of $t^{(q / \bar{s})(\beta)}$, but for some $\eta \epsilon^{<\mathrm{ht}\left(t^{z(\beta)}\right)} 2$ which is not in $t^{z(\beta)}$, we have

$$
\left(z \lceil \beta ) \oplus ( q _ { l } / \overline { s } ) \left\lceil\beta \Vdash " \eta \in \mathcal{T}_{\sim}^{q_{l} / \bar{s}(\beta)} \cup \mathcal{T}^{z(\beta)} " .\right.\right.
$$

(iii) or $t^{q / \bar{s}(\beta)}$ is an end extension of $t^{z(\beta)}$, but for some $\eta \in^{<\mathrm{ht}\left(t^{q(\beta)}\right)} 2$ which is not in $t^{q(\beta)}$ we have

$$
\left(z\lceil\beta) \oplus\left(q_{l} / \bar{s}\right) \uparrow \beta \Vdash " \eta \in \mathcal{T}_{\sim}^{q_{l} / \bar{s}(\beta)} \cup \mathcal{\sim}^{z(\beta)} " .\right.
$$

When choosing $s_{n}$, for some large enough $n$ we have asked if there is $z \in P_{\alpha}^{\prime}$ with $\operatorname{Dom}(z)=\operatorname{Dom}_{s_{0}\lceil\alpha}^{*}(r) \cap u$ and such that 
(A) $z \uparrow \beta \geq\left(r / s_{n}\right) \uparrow \beta$

(B) $(\forall \gamma \in \operatorname{Dom}(z))\left(\exists t_{\gamma}\right)\left(\Vdash_{P_{\gamma}} “{\underset{\sim}{z}}^{z(\gamma)}=t_{\gamma}\right.$ ”).

(C) For all $l \leq n$ one of the following happens

(a) $z \uparrow \beta$ is incompatible with $\left(q_{l} / s_{n}\right) \uparrow \beta$,

(b) Neither $t^{z(\beta)}$ is an end extension of $t^{q_{l}(\beta)}$, nor is $t^{q_{l}(\beta)}$ end extension of $t^{z(\beta)}$.

(c) $t^{z(\beta)}$ is an end extension of $t^{q_{l}(\beta)}$, but for some $\eta \epsilon^{<\mathrm{ht}\left(t^{z(\beta)}\right)} 2$ which is not in $t^{z(\beta)}$, we have

$$
(z \uparrow \beta) \oplus\left(q_{l} / s_{n}\right) \uparrow \beta \Vdash " \eta \in{\underset{\sim}{\mathcal{T}}}^{q_{l} / s_{n}(\beta)} \cup \underset{\sim}{\mathcal{T}} z(\beta) " .
$$

(d) $t^{q_{l}(\beta)}$ is an end extension of $t^{z(\beta)}$, but for some $\eta \in<\operatorname{ht}\left(t^{q(\beta)}\right) 2$ which is not in $t^{q_{l}(\beta)}$ we have

$$
(z \uparrow \beta) \oplus\left(q_{l} / s_{n}\right) \uparrow \beta \Vdash " \eta \in{\underset{\sim}{\mathcal{T}}}^{q_{l} / s_{n}(\beta)} \cup{\underset{\sim}{\mathcal{T}}}^{z(\beta)} "
$$

If after some $n^{*}$ the answer to the above question was never positive, this means that $z^{*}$ could not have been used as a witness, which means that $z^{*}$ is compatible with some $q_{l} / \bar{s}$.

Suppose that the answer was positive at some large enough $n$, and let this be exemplified by some $z$. Without loss of generality we have $z \in M$. We can find $m>n$ such that with $q_{m}$ in place of $q_{l}$ above, neither of the first two possibilities happen. So suppose the third one does. Hence for some $k<m$ we have that

$$
z \uparrow \beta+\left(q_{m}+s_{k}\right) \uparrow \beta \Vdash " \eta \in{\underset{\sim}{\mathcal{T}}}^{q_{m}(\beta)} \cup{\underset{\sim}{\mathcal{T}}}^{z(\beta)} "
$$

for some $\eta \in \operatorname{ht}^{\left(t^{z(\beta)}\right)} 2 \backslash t^{z(\beta)}$. But this is a contradiction with $z \uparrow \beta$ being compatible with $q_{m}+s_{k}$.

$(4)^{\alpha}$ Similar.

$(5)^{\alpha}$ For $l<\omega$ let

$$
\begin{array}{r}
\Upsilon_{l}^{*} \stackrel{\text { def }}{=} \operatorname{type}_{\bar{x}}^{s_{0} \uparrow(\alpha \cap E V E N), n}\left(\left(r+s_{l}\right) \uparrow(\alpha \cap E V E N)\right) \text { and } \\
\Upsilon_{l}^{\prime} \stackrel{\text { def }}{=} \operatorname{type}_{\bar{x}: p_{l}\lceil(\alpha \cap E V E N)}^{p_{l} \uparrow(\alpha \cap E V E N), n}\left(\left(r / \bar{s}+p_{l}\right) \uparrow(\alpha \cap E V E N)\right) .
\end{array}
$$


We show that for large enough $l$ we have $\Upsilon_{l}^{*}=\Upsilon_{l}^{\prime}$, by comparing the corresponding 9 coordinates. It is easy to see that for any $l$ we have that

$$
\begin{aligned}
& n^{\left[\Upsilon_{l}^{*}\right]}=n^{\left[\Upsilon_{l}^{\prime}\right]}=n, o^{\left[\Upsilon_{l}^{*}\right]}=o^{\left[\Upsilon_{l}^{\prime}\right]}=1, \beta^{\left[\Upsilon_{l}^{*}\right]}=\beta^{\left[\Upsilon_{l}^{\prime}\right]}=\beta, \\
& t^{\left[\Upsilon_{l}^{*}\right]}=t^{\left[\Upsilon_{l}^{\prime}\right]}=t^{r(\beta)} \text { and } \\
& \bar{\epsilon}^{\left[\Upsilon_{l}^{*}\right]}=\bar{\epsilon}^{\left[\Upsilon_{l}^{\prime}\right]}=\operatorname{Dom}(r / \bar{s}) \cap \alpha \text { in the increasing enumeration. }
\end{aligned}
$$

We now prove that for large enough $l$ we have $w^{\left[\Upsilon_{l}^{*}\right]}=w^{\left[\Upsilon_{l}^{\prime}\right]}$. Given $m<n$.

When choosing $s_{l}$ 's, we have infinitely often asked if there is $s^{\prime} \geq_{\mathrm{pr}} s_{l}$ and $q \geq r$ such that

(i) $s^{\prime} \in G R_{s_{0}, u}$,

(ii) $\left(q+s^{\prime}\right) \uparrow(\beta \cap E V E N) \in \in^{\bar{x}} A_{m}^{\beta}$

(iii) For some $l^{\prime} \geq l$ we have $\left(q / s^{\prime}\right) \uparrow(\beta \cap E V E N) \in \in^{\bar{x}: p_{l} \uparrow(\beta \cap E V E N)} A_{m}^{\beta}$, and if possible, we have chosen some such $s^{\prime}$ as $s_{l+1}$.

$\underline{\text { Possibility } 1}$. For some $l$ large enough we chose $s_{l+1}$ to satisfy $(i)-($ iii $)$ above with $s_{l+1}$ in place of $s^{\prime}$.

Hence there is $q$ which witnesses the choice. By $(2)^{\alpha}$, we have $q / \bar{s} \geq r / \bar{s}$, so $m \in w^{\left[\Upsilon_{l}^{*}\right]} \cap w^{\left[\Upsilon_{l}^{\prime}\right]}$.

Possibility 2. For no large enough $l$ could we have chosen $s_{l+1}$ so to satisfy $(i)-($ iii $)$ above with $s_{l+1}$ in place of $s^{\prime}$.

Suppose that $l$ is large enough and $m \in w^{\left[\Upsilon_{l}^{*}\right]}$, as exemplified by $q$. Without loss of generality $q \in M$. Hence $q \geq$ apr $\left(r+s_{l}\right) \uparrow(\beta \cap E V E N)$ and $q \in R_{s_{0} \uparrow(\beta \cap E V E N)}^{+}$. We have $(q / \bar{s}) \uparrow \beta \geq(r / \bar{s}) \uparrow \beta$. Let $q, i$ be such that ${ }^{\bar{x}} A_{m}^{\beta}=z / E_{\bar{x}}^{s_{0}}{ }^{(\beta \cap E V E N), i}$. Without loss of generality we have $z \in M$. Hence, by the induction hypothesis we have

$$
\bar{x}: p_{l} \uparrow(\beta \cap E V E N) A_{m}^{\beta}=\left(z / \bar{s}+p_{l}\right) \uparrow(\beta \cap E V E N) / E_{\bar{x}: p_{l}}^{p_{l} \uparrow(\beta \cap E V E N), i} .
$$

By the induction hypothesis, for large enough $l$ we have $q / \bar{s} \in \bar{x}: p_{l} A_{m}^{\beta}$. This is a contradiction. Hence $m \notin w^{\left[\Upsilon_{l}^{*}\right]}$ for all large enough $l$. 
We similarly show that $m \notin w^{\left[\Upsilon_{l}^{\prime}\right]}$ for all large enough $l$.

Other parts of the claim are checked similarly.

$\star 7.11$

\section{Obtaining \&s in $V^{P}$}

Claim 8.1. $V^{P} \models$ o.

Proof of the Claim.

Definition 8.2. Suppose that $\bar{N}$ is a sequence of elementary submodels of $\left\langle H(\chi), \in,<_{\chi}^{*}\right\rangle$ and $\bar{a}$ a finite sequence in $\bar{N}(0)$. We say that an $x \in H(\chi)$ is chosen canonically for $(\bar{N}, \bar{a})$, if the choice of $x$ depends only on the isomorphism type of $(\bar{N}, \bar{a})$ as a submodel of $\left(H(\chi), \in,<_{\chi}^{*}, \vec{a}\right)$, where $\vec{a}$ is a finite list of constant symbols (interpreted in $\bar{N}(0)$ as $\bar{a}$ ).

Main Claim 8.3. (1) Given a sequence $\bar{N}=\left\langle N_{n}: n<\omega\right\rangle$ of countable elementary submodels of $\left\langle H(\chi), \in,<_{\chi}^{*}\right\rangle$ with $N_{n} \in N_{n+1}$ for all $n$, and $\bar{Q}, \bar{Q}^{\prime}, \tau \in N_{0}$ and $p \in N_{0} \cap P$ such that

$$
p \Vdash " \tau \in\left[\omega_{1}\right]^{\aleph_{1}} " .
$$

Let $\bar{a}=\left\langle\tau, p, \bar{Q}, \bar{Q}^{\prime}\right\rangle$ and let $\delta \stackrel{\text { def }}{=} \bigcup_{n<\omega}\left(N_{n} \cap \omega_{1}\right)$.

Then there is

(a) a strictly increasing sequence $\bar{\beta}=\bar{\beta}(\bar{N}, \bar{a})=\left\langle\beta_{n}: n<\omega\right\rangle$ with $\sup _{n<\omega} \beta_{n}=\delta$, which is chosen canonically for $(\bar{N}, \bar{a})$ and

(b) a condition $r^{\oplus}=r_{\bar{N}, \bar{a}}^{\oplus} \geq p$, with $r^{\oplus} \Vdash "\left\{\beta_{n}: n<\omega\right\} \subseteq \tau_{\sim}$ ".

(2) Values of $\beta_{n} \stackrel{\text { def }}{=} \bar{\beta}(\bar{N}, \bar{a})(n)$ for $n<\omega$, and the fact that there is an $r^{\oplus} \geq p$ such that $r^{\oplus} \Vdash$ " $\left\{\beta_{n}: n<\omega\right\} \subseteq \tau$ " only depend on the isomorphism type of $(\bar{N}, \bar{a})$ as a submodel of $\left(H(\chi), \in,<_{\chi}^{*}, \vec{a}\right)$.

Proof of the Main Claim. (1).

Let $N_{\omega} \stackrel{\text { def }}{=} \bigcup_{n<\omega} N_{n}$. 
Subclaim 8.4. Suppose that $\bar{N}$ and $\bar{a}$ are as in the statement of the Main Claim 8.3 and $N_{\omega}$ as defined above. Let $\xi_{\bar{N}} \stackrel{\text { def }}{=} \operatorname{otp}\left(N_{\omega} \cap \omega_{2} \cap O D D\right)$ and let $h$ be the order isomorphism exemplifying this. Let $\left\langle u_{n}^{*}: n<\omega\right\rangle$ be the $<_{\chi}^{*}$-first increasing sequence of finite sets such that $u_{n} \stackrel{\text { def }}{=} h^{-1}\left(u_{n}^{*}\right) \subseteq N_{n}$ and $\bigcup_{n<\omega} u_{n}^{*}=\xi_{\bar{N}}$. Let $\left\{\varphi_{n}: n<\omega\right\}$ be the $<_{\chi}^{*}$-first enumeration of the first order formulas with parameters in $N_{\omega}$, each formula appearing infinitely often, and such that the parameters of $\varphi_{n}$ are contained in $N_{n}$.

Then there are sequences

$$
\bar{p}=\bar{p}_{\bar{N}, \bar{a}}=\left\langle p_{n}: n<\omega\right\rangle \text { and } \bar{q}=\bar{q}_{\bar{N}, \bar{a}}\left\langle q_{n}: n<\omega\right\rangle
$$

chosen canonically for $\bar{N}$ and $\bar{a}$ such that

(i) $q_{0}=p_{0}=p$.

(ii) $p_{n+1} \geq_{\text {pr }} p_{n}$.

(iii) $p_{n} \leq_{\text {apr }} q_{n}$.

(iv) $p_{n}, q_{n} \in N_{n+1}$.

(v) For all $n$ and $\alpha \in u_{n}$, we have that

$$
p_{n+1}\left\lceil\alpha \Vdash{ }_{\sim}^{f} p^{p_{n+1}(\alpha)}(x)<\underset{\sim}{f}{ }^{p_{n}(\alpha)}\left(x \uparrow\left(\delta^{*}\left(p_{n}\right)+1\right)+1 / 2^{n}\right. \text {, }\right.
$$

for all $x \in \underset{\sim}{\underset{\sim}{g_{\alpha}-1}(n)} \underset{\delta^{*}\left(p_{n+1}\right)}{\alpha}$.

(vi) For every $n$

either

( $\alpha$ ) There is no $p^{\prime} \geq_{\text {pr }} p_{n}$ and $q^{\prime} \geq_{\text {apr }} p^{\prime}$ such that $\varphi_{n}\left(p^{\prime}, q\right)$ and (v) above holds with $p^{\prime}$ in place of $p_{n+1}$,

or

( $\beta)\left(p_{n}^{\prime}, q_{n}\right)$ are the $<_{\chi}^{*}$-first elements of $H(\chi)$ which exemplify that $(\alpha)$ does not happen, with $p_{n}^{\prime}$ in place of $p^{\prime}$ and $q_{n}$ in place of $q$. 
Proof of the Subclaim. The proof is straightforward. Construct $p_{n}, q_{n}$ by induction on $n$, the step at the stage $n=0$ being given. At the stage $n+1$, we are given $p_{n}$ and we consider $\varphi_{n}$. If option $(\alpha)$ holds, just let $p_{n+1}=q_{n+1} \stackrel{\text { def }}{=} p_{n}$. If $(\beta)$ holds, then find $\left(p_{n+1}, q_{n+1}\right)$ as described in $(\beta)$, and note that $p_{n+1}, q_{n+1} \in N_{n+2} . \star 8.4$

Subclaim 8.5. Suppose that $\bar{N}, \bar{a}$ and $\bar{p}=\bar{p}_{\bar{N}, \bar{a}}$ are as in the Claim 8.4.

Then there is a canonically chosen condition $p_{\omega}=p_{\bar{N}, \bar{a}}$ such that for all $n$ we have $p_{n} \leq_{\text {pr }} p_{\omega}$, while $\operatorname{Dom}\left(p_{\omega}\right)=N_{\omega} \cap \omega_{2}$ and $\delta^{*}\left(p_{\omega}\right)=N_{\omega} \cap \omega_{1}$.

Proof of the Subclaim. The same argument as the one used in Claim 5.1 to prove $(2)^{\alpha}$ at the stages $\alpha$ of countable cofinality. $\star$. .5

There is $r \geq p$ such that $r \Vdash$ " $\beta \in \mathcal{\sim}$ " for some $\beta>\delta$. By Observation 4.13 and Claim 6.2 there are $s_{0}, r^{*}$ such that

(i) $p_{\omega} \leq_{\text {pr }} s_{0} \leq_{\text {apr }}^{+} r^{*}$, and

(ii) $\left[\alpha \in \operatorname{Dom}\left(r^{*}\right) \& \neg\left(r^{*} \mid \alpha^{\prime \prime} \Vdash r^{*}(\alpha)=s_{0}(\alpha) "\right)\right] \Longrightarrow \alpha \in \operatorname{Dom}\left(p_{\omega}\right)$.

(iii) For some $\beta^{*}>\delta$ we have $r^{*} \Vdash " \beta^{*} \in \tau_{\sim}$ ".

Now let $M$ be countable $\prec\left\langle H(\chi), \in,<_{\chi}^{*}\right\rangle$ such that $\left\{\bar{N}, s_{0}, r^{*}, \beta^{*}\right\} \subseteq M$.

Let $v \stackrel{\text { def }}{=}\left\{\alpha: \neg\left(r^{*} \mid \alpha \Vdash " r^{*}(\alpha)=s_{0}(\alpha)\right.\right.$ ") $\}$, hence $v$ is finite $\subseteq \operatorname{Dom}\left(p_{\omega}\right)$.

Let $\bar{s}=\left\langle s_{n}: n<\omega\right\rangle$ be a generic enough sequence for $\left(\mathrm{GR}_{s_{0}, \operatorname{Dom}\left(p_{\omega}\right)}, M\right)$. Let $\bar{x}$ be a canonical assignment for $s_{0} \uparrow E V E N$.

Definition of $\bar{\beta}$ and $r^{\oplus}$.

By induction on $n<\omega$ we shall define $\beta_{n}$, as well as natural numbers $m_{n}$ and conditions $r_{n}$.

$\underline{n=0}$. We let $m_{0}=n_{0}$ and $\beta_{0} \stackrel{\text { def }}{=} N_{m_{0}} \cap \omega_{1}$.

$n+1$. Given is $m_{n}$ and $\beta_{n}$.

Let $m^{\prime} \stackrel{\text { def }}{=} m_{n+1}^{\prime}$ be the first large enough integer $>m_{n}$ so that $\operatorname{type}_{\bar{x}}^{s_{0}\lceil E V E N, n}\left(\left(r^{*}+s_{m^{\prime}}\right)\lceil E V E N)=\operatorname{type}_{\bar{x}: p_{m^{\prime}}}^{p_{m^{\prime}} \uparrow E V E N, n}\left(\left(r^{*} / \bar{s}+p_{m^{\prime}}\right) \mid E V E N\right) \stackrel{\text { def }}{=} \Upsilon_{n}\right.$.

We now consider the formula $\psi^{n}\left(x_{0}, x_{1}\right)$ saying that 
(I) $p_{m^{\prime}} \leq_{\text {pr }} x_{0} \leq_{\text {apr }} x_{1}$ and

(II) $x_{1} \Vdash ~ " \gamma \in \tau$ " for some $\gamma>\left\{N_{m^{\prime}} \cap \omega_{1}\right\}$ and

(III) We have type $\bar{x}_{\bar{x}: p_{m^{\prime}}}^{x_{0} \vdash E V E N, n}\left(x_{1} \uparrow E V E N\right)=\Upsilon_{n}$.

Let $m_{n+1}$ be the first $m>m^{\prime}$ such that $\varphi_{m}=\psi_{n}$. Hence we have chosen $\left(p_{m_{n+1}+1}, q_{m_{n+1}+1}\right)$ so that $\psi_{n}\left(\left(p_{m_{n+1}+1}, q_{m_{n+1}+1}\right)\right)$ holds, as is exemplified by $\left(s_{0}, r^{*}+s_{m}\right)$.

Let $r_{n} \stackrel{\text { def }}{=}\left(q_{m_{n}}+p_{\omega}\right) \uparrow E V E N$, for $n<\omega$. We shall define $r^{\oplus}$ so that $r^{\oplus} \geq r_{n}$ for all $n$. Hence $r^{\oplus} \Vdash "\left\{\beta_{n}: n<\omega\right\} \subseteq \tau_{\sim}$ ".

The Main Point

Why does such $r^{\oplus}$ exist? All $r_{n}$ are elements of $R_{p_{\omega}}^{+}$, and by the definition of $\Upsilon_{n}$, each has the property that $r_{n} E_{\bar{x}: p_{\omega}}^{p_{\omega}, n}\left(\left(r^{*} / \bar{s}+p_{\omega}\right)\lceil E V E N)\right.$. By Lemma 2.13, there must be $\check{r} \in R_{p_{\omega}}$ which is a common upper bound to $\left\{r_{n}: n<\omega\right\}$. Let $r \stackrel{\text { def }}{=} \check{r}+p_{\omega}$.

Proof of the Main Claim continued.

(2) It suffices to observe the following

Observation 8.6. Given $\bar{N}, \bar{a}$ as in Main Claim 8.3. Let $\bar{p}$ and $\bar{q}$ be as in Subclaim 8.4. Let $\bar{z}$ be a canonical assignment for $p_{\omega} \mid E V E N$. Suppose that $X \in[\omega]^{\aleph_{0}}$ is such that $\left\{q_{n}\lceil E V E N: n \in X\}\right.$ has an upper bound in $\mathfrak{B}_{p_{\omega}, \bar{z}}$. Suppose that $f:(\bar{N}, \bar{a}) \rightarrow\left(\bar{N}^{\prime}, \bar{a}^{\prime}\right)$ is an isomorphism.

Then

$\left\{f\left(q_{n}\lceil E V E N): n \in X\right\}\right.$ has an upper bound in $\mathfrak{B}_{n<\omega} f\left(p_{n}\right), \bigcup_{n<\omega} f\left(\bar{z}: p_{n}\right)$.

$\star 8.3$

Now we can finish proving Claim 8.1 and so Theorem 3.1. Let

$$
\mathfrak{A} \stackrel{\text { def }}{=}\left(H(\chi), \in,<_{\chi}^{*}, p, \tau, \bar{Q}, \bar{Q}^{\prime}\right),
$$

where $p, \tau, \bar{Q}, \bar{Q}^{\prime}$ are constant symbols. We arrange $\diamond$ in $V$ in this form:

There is a sequence

$$
\left\langle\bar{N}^{\delta}=\left\langle N_{i}^{\delta}: i<\delta\right\rangle: \delta<\omega_{1} \text { limit }\right\rangle
$$

such that 
1. $N_{i}^{\delta}$ is a countable elementary submodel of $\mathfrak{A}$, with $N_{i}^{\delta} \cap \omega_{1}<\delta$, $\bar{N}^{\delta} \uparrow i \in N_{i+1}^{\delta}$.

2. $\bar{N}^{\delta}$ is continuously increasing.

3. For every continuously increasing sequence $\left\langle N_{i}: i<\omega_{1}\right\rangle$ of countable elementary submodels of $\mathfrak{A}$, there is a stationary set of $\delta$ such that the isomorphism type of $\left\langle N_{i}: i<\delta\right\rangle$ is the same as that of $\left\langle N_{i}^{\delta}: i<\delta\right\rangle$.

For $\delta<\omega_{1}$ a limit ordinal, we choose the $<_{\chi}^{*}$-first increasing $\omega$-sequence $\left\langle\epsilon_{n}^{\delta}: n<\omega\right\rangle$ of ordinals such that $\sup _{n<\omega} \epsilon_{n}^{\delta}=\delta$ and $\epsilon_{0}^{\delta}=0$. We define sets $A_{\delta}$ for such $\delta$ as follows. Let $N^{\delta} \stackrel{\text { def }}{=} \bigcup_{i<\delta} N_{i}^{\delta}$.

$$
\begin{gathered}
\text { If } N^{\delta} \cap \omega_{1}=\delta, p^{N_{0}^{\delta}} \in P^{\prime} \cap N_{0}^{\delta} \text { and } p^{N_{0}^{\delta} \Vdash} " \mathcal{\sim}^{\tau_{0}^{\delta}} \in\left[\omega_{1}\right]^{\aleph_{1}} \text { ", then } \\
A_{\delta} \stackrel{\text { def }}{=} \operatorname{Rang}\left(\bar{\beta}\left(\left\langle N_{\epsilon_{n}^{\delta}}^{\delta}: n<\omega\right\rangle\right)\right) .
\end{gathered}
$$

Otherwise, we let $A_{\delta}$ be the range of any cofinal $\omega$-sequence in $\delta$.

We claim that $\left\langle A_{\delta}: \delta\right.$ limit $\left\langle\omega_{1}\right\rangle$ is a $\mathbf{p}$-sequence in $V^{P}$.

So suppose that $p^{*} \Vdash$ " $\tau^{*} \in\left[\omega_{1}\right]^{\aleph_{1}}$ " and $p \in P^{\prime}$. We fix a continuously increasing sequence $\bar{N}=\left\langle N_{i}: i<\omega_{1}\right\rangle$ of countable elementary submodels of $\mathfrak{A}$ such that $\bar{Q}=\bar{Q}^{N_{0}}, p^{N_{0}}=p^{*}, \tau_{\sim}^{N_{0}}=\underset{\sim}{\tau}, \bar{Q}^{\prime}=\left[\bar{Q}^{\prime}\right]^{N_{0}}$ and $\bar{N} \uparrow i \in N_{i+1}$ for all $i<\omega_{1}$. Then

$$
C \stackrel{\text { def }}{=}\left\{\delta<\omega_{1}: \delta \text { limit and } N_{\delta} \cap \omega_{1}=\delta\right\}
$$

is a club of $\omega_{1}$. Hence there is $\delta<\omega_{1}$ such that $\left\langle N_{i}: i<\delta\right\rangle$ and $\left\langle N_{i}^{\delta}: i<\delta\right\rangle$ have the same isomorphism type. So $A_{\delta}$ is defined by the first clause in its definition.

Hence, by Main Claim $8.3(2)$, we have $A_{\delta}=\operatorname{Rang}\left(\bar{\beta}\left(\left\langle N_{\epsilon_{n}^{\delta}}: n<\omega\right\rangle\right)\right)$, while $\bar{r}(\bar{N} \uparrow \delta)$ has an upper bound, say $r^{\otimes}$. Now $r^{\otimes} \geq p$ and $r^{\otimes} \Vdash$ " $A_{\delta} \subseteq \tau_{\sim}^{*}$ ". $\star 8.1$

$\star 3.1$

Remark 8.7. Note that the club sequence $\left\langle A_{\delta}: \delta<\omega_{1}\right\rangle$ we obtained for the final model, is in fact a sequence in $V$. 


\section{References}

[BMR] J. Baumgartner, J. Malitz, and W. Reindhart, Embedding Trees in the Rationals, Proceedings of the National Academy of Sciences, vol. 67, N0. 4, pp. 1748-1753, December 1970.

[DjSh 574] M. Džamonja and S. Shelah, Similar but not the same: various versions of $\mathbf{\$}$ do not coincide, to appear in the Journal of Symbolic Logic.

[FShS 544] S. Fuchino, S. Shelah and L. Soukup, Sticks and clubs, to appear in the Annals of Pure and Applied Logic.

[Je] R. B. Jensen, The Fine Structure of the Constructible Hierarchy, Annals of Mathematical Logic (APAL), 1972 (4), pp. 229-308.

[Ko] P. Komjáth, Set systems with finite chromatic number, European Journal of Combinatorics, 1989 (10), pp. 543-549.

[KuVa] K. Kunen and J. Vaughan (eds.), Handbook of Set Theoretic Topology, North-Holland, 1984.

[Mi] A. W. Miller, Arnie Miller's problem list, Proceedings of Set theory of the reals (Ramat Gan 1991), Israel Mathematical Conferences Proceedings vol. 6, pp. 645-654, Bar Ilan University 1993.

[Ost] A. J. Ostaszewski, On countably compact perfectly normal spaces, Journal of London Mathematical Society, 1975 (2) 14, pp. 505516.

[Sh -f] S. Shelah, Proper and Improper Forcing, Springer, Perspectives in Mathematical Logic, 1998.

[Sh 98] S. Shelah, Whitehead groups may not be free, even assuming CH, II, Israel Journal of Mathematics, 1980 (35) pp. 257-285.

[Sh 176] S. Shelah, Can you take Solovay's inaccessible away?, Israel Journal of Mathematics, 1984 (48), pp.1-47. 
[Tr] J. Truss, Sets having calibre $\aleph_{1}$, Logic Coll. 76, Oxford, 1976, pp. 595-612, Studies in Logic and Foundations of Mathematics, Vol. 87, North-Holland, 1977. 